\title{
Upregulation of miR-132-3p in cholangiocarcinoma tissues: A study based on RT-qPCR, The Cancer Genome Atlas miRNA sequencing, Gene Expression Omnibus microarray data and bioinformatics analyses
}

\author{
HUA-YU WU ${ }^{1,2^{*}}$, SHUANG XIA ${ }^{3 *}$, AN-GUI LIU ${ }^{1}$, MIN-DA WEI $^{1}$, ZHONG-BIAO CHEN $^{4}$, \\ YU-XIN LI ${ }^{1}$, YU HE ${ }^{1}$, MIN-JUN LIAO ${ }^{1}$, QI-PING HU ${ }^{2}$ and SHANG-LING PAN ${ }^{1}$ \\ Departments of ${ }^{1}$ Pathophysiology, ${ }^{2}$ Cell Biology and Genetics, and ${ }^{3}$ Human Anatomy, School of Pre-clinical Medicine, \\ Guangxi Medical University; ${ }^{4}$ Department of Pathology, First Affiliated Hospital of Guangxi Medical University, \\ Nanning, Guangxi Zhuang Autonomous Region 530021, P.R. China
}

Received November 12, 2018; Accepted April 5, 2019

DOI: $10.3892 / \mathrm{mmr} .2019 .10730$

\begin{abstract}
MicroRNAs (miRNAs/miRs) have been reported to be closely associated with numerous human diseases, including cholangiocarcinoma (CCA). However, the number of miRNAs known to be involved in CCA is limited, and the association between miR-132-3p and CCA remains unknown. In the present study, the clinical role of miR-132-3p and its potential signaling pathways were investigated by multiple approaches. Reverse transcription-quantitative PCR (RT-qPCR), CCA-associated Gene Expression Omnibus (GEO), ArrayExpress and Sequence Read Archive(SRA) miRNA-microarray ormiRNA-sequencing data were screened, and meta-analyses were conducted, in order to calculate the receiver operating characteristic (ROC) curve and standardized mean difference (SMD). The predicted target genes of miR-132-3p were obtained from 12 online databases and were combined with the downregulated differentially expressed genes identified in the RNA-sequencing data of CCA. Gene Ontology annotation and pathway analysis were
\end{abstract}

Correspondence to: Professor Shang-Ling Pan, Department of Pathophysiology, School of Pre-clinical Medicine, Guangxi Medical University, 22 Shuangyong Road, Nanning, Guangxi Zhuang Autonomous Region 530021, P.R. China

E-mail: slpan@gxmu.edu.cn

Professor Qi-Ping Hu, Department of Cell Biology and Genetics, School of Pre-clinical Medicine, Guangxi Medical University, 22 Shuangyong Road, Nanning, Guangxi Zhuang Autonomous Region 530021, P.R. China

E-mail: huqiping@gxmu.edu.cn

*Contributed equally

Key words: miR-132-3p, cholangiocarcinoma, reverse transcriptionquantitative PCR, miRNA sequencing, Gene Expression Omnibus, microarray performed in WebGestalt. Protein-protein interaction analyses were conducted in STRING. The Cancer Genome Atlas (TCGA) mRNA expression profiles were used to validate the expression levels of hub genes at the mRNA level. The Human Protein Atlas was used to identify the protein expression levels of hub genes in CCA tissues and non-tumor biliary epithelium. The meta-analyses comprised 10 groups of RT-qPCR data, eight GEO microarray datasets and one TCGA miRNA-sequencing dataset. The SMD of miR-132-3p in CCA was $0.75(95 \%$ CI: $0.25,1.24)$, which indicated that miR-132-3p was overexpressed in CCA tissues. This finding was supported by a summary ROC value of 0.80 (95\% CI: $0.76,0.83)$. The pooled sensitivity and specificity were $0.81(95 \% \mathrm{CI}: 0.59,0.93)$ and 0.71 (95\% CI: $0.58,0.81)$, respectively. The relative expression level of miR-132-3p in the early stage of CCA (stages I-II) was $6.8754 \pm 0.5279$, which was markedly lower than that in the advanced stage (stages III-IVB), $7.3034 \pm 0.3267$ ( $\mathrm{P}=0.003)$. Consistently, the miR-132-3p level in low-grade CCA (grades G1-G2) was 6.7581 \pm 0.5297 , whereas it was $7.1191 \pm 0.4651$ in patients with high-grade CCA (grades G3-G4) $(\mathrm{P}=0.037)$. Furthermore, 555 potential target genes of miR-132-3p in CCA were mainly enriched in the 'Focal Adhesion-PI3K-Akt-mTOR -signaling pathway'. In conclusion, upregulation of miR-132-3p may serve a pivotal role in the tumorigenesis and progression of CCA by targeting different pathways. Further in vitro and in vivo studies are required to support the current findings.

\section{Introduction}

Cholangiocarcinoma (CCA) is a malignant tumor derived from the intrahepatic and extrahepatic bile duct $(1,2)$. At present, CCA is the most common hepatic and bile duct-associated malignancy with poor prognosis besides hepatocellular carcinoma (3-6). The rates of all types of CCA have been increasing in the last few decades (7). However, the molecular mechanism of CCA remains to be elucidated, and efficient therapeutic options are lacking, which leads to the urgency of exploring specific biomarkers and effective therapies for CCA. 
Among all possible molecular events involved in the tumorigenesis and development of CCA, microRNAs (miRNAs/miRs) have been documented to serve essential roles. As small noncoding single-stranded RNA molecules, miRNAs participate in biological processes by functioning as post-transcriptional regulatory factors (8-12). In recent years, miRNAs have been reported to be closely associated with numerous human diseases, including CCA (13). Previous studies have reported that several miRNAs may regulate the proliferation, invasion and metastasis of CCA, and may be potential biomarkers used to evaluate the progression and prognosis of patients with CCA (5-7,14-16). However, the number of known miRNAs involved in CCA is limited and the majority of studies conducted to date have focused on a small sample size of clinical specimens. Therefore, it is necessary to identify novel miRNA candidates and explore the clinical value of these miRNAs in a larger sample size.

miR-132-3p has been reported to be differentially expressed in several cancer types, and to participate in the formation and metastasis of several malignant tumors. miR-132-3p has been associated with the post-transcriptional regulation of BCRP/ABCG2 in renal cell carcinoma $(8,17)$. Overexpressed miR-132-3p can regulate executioner caspase-7 in pancreatic ductal adenocarcinoma and may contribute to malignant progression (18). Furthermore, miR-132-3p is significantly decreased in gastric cancer tissues compared with in peripheral non-cancer gastric tissues, and its alteration may serve as a novel molecular marker for gastric cancer (19). However, the association between miR-132-3p and CCA, and its clinical significance, remain to be investigated. A limited number of genes have been identified as targets of miR-132-3p in various diseases. For instance, Cai et al (20) reported that miR-132-3p regulates BCL2L11 in tuberous sclerosis complex angiomyolipoma, whereas Zhou et al (21) reported that miR-132-3p regulates ADAMTS-5 expression and promotes chondrogenic differentiation in rat mesenchymal stem cells. It is well known that a single miRNA can have multiple targets. However, the targets and signaling pathways of miR-132-3p in CCA remain unknown.

Our preliminary work mining miRNA microarray and miRNA-sequencing data of CCA revealed several aberrantly expressed miRNAs (Wu et al, unpublished data), and miR-132-3p was among them. Therefore, to identify the role of miR-132-3p in CCA, the present study reprocessed the expression profiles of miR-132-3p in CCA and non-tumor tissues from microarray and miRNA-sequencing data. Furthermore, meta-analyses were performed to evaluate the expression levels of miR-132-3p in CCA based on all available cases. Subsequently, the potential role of miR-132-3p as a biomarker for predicting prognosis in CCA was investigated. In order to explore the molecular function of miR-132-3p in CCA, Gene Ontology (GO) and WikiPathway analyses were performed to investigate the prospective targets of miR-132-3p. Finally, the mRNA and protein expression levels of hub target genes were verified.

\section{Materials and methods}

Collection of clinical samples and reverse transcription-quantitative PCR (RT-qPCR). Samples were collected from patients with CCA from the First Affiliated Hospital, Guangxi Medical University between December 2010 and September 2017. In total, 25 CCA and 22 paracarcinoma bile duct tissues were collected, which included 16 males and 9 females with the ages ranging from 34 to 65 years old. In three patients, paracarcinoma bile duct tissue was not collected. This study was approved by the Ethics Committee of First Affiliated Hospital, Guangxi Medical University, China. Informed written consent was obtained from all patients participating in the study. Total RNA from fixed (overnight with $10 \%$ neutral-buffered formalin at $25^{\circ} \mathrm{C}$ ) and paraffin embedded samples was extracted from sections using the miRNeasy FFPE kit (Qiagen $\mathrm{GmbH}$ ). For RT into cDNA, the All-in-One ${ }^{\mathrm{TM}}$ miRNA First-Strand cDNA Synthesis kit (QP013, GeneCopoeia, Inc.) was used to transcribe $10 \mu \mathrm{l}$ purified RNA. The prepared reaction mixture was gently mixed, incubated at $37^{\circ} \mathrm{C}$ for $60 \mathrm{~min}$ after brief centrifugation, followed by incubation at $85^{\circ} \mathrm{C}$ for $5 \mathrm{~min}$ for RT. qPCR, using miR-specific primers and universal adaptor PCR primers purchased from GeneCopoeia, Inc. with confidential sequences, was performed using the Applied Biosystems 7500 Real-Time PCR system (cat. no. QP010; Applied Biosystems; Thermo Fisher Scientific, Inc.). The All-in-one miRNA qPCR kit was used for the qPCR (cat. no. QP013; GeneCopoeia, Inc.). The reactions were incubated in a 96 -well plate at $95^{\circ} \mathrm{C}$ for $10 \mathrm{~min}$, followed by 40 cycles at $95^{\circ} \mathrm{C}$ for $10 \mathrm{sec}, 60^{\circ} \mathrm{C}$ for $20 \mathrm{sec}$ and $72^{\circ} \mathrm{C}$ for $34 \mathrm{sec}$, fluorescent signals were measured during the extension phase. The results were normalized to the reference of U6RNA and calculated using the $2^{-\Delta \Delta \mathrm{Cq}}$ method (22). All reactions were run in triplicate.

Collection and analysis of miRNA-microarray and miRNA-sequencing data of CCA. The flowchart of the present study is shown in Fig. 1. To find potential datasets of interest, CCA-associated Gene Expression Omnibus (GEO; https://www.ncbi.nlm.nih.gov/geo/), ArrayExpress (https://www.ebi.ac.uk/arrayexpress/) and Sequence Read Archive (SRA; https://www.ncbi.nlm.nih.gov/sra/) (23-25) microarray or miRNA-sequencing data were used with the following search strategies: ('bile duct' OR 'cholangiocellular') AND ('cancer' OR 'carcinoma' OR 'tumor' OR 'neoplas*' OR 'malignan*' OR 'adenocarcinoma' OR 'cholangiocarcinoma'). The screening criteria were as follows: Firstly, the samples in each dataset must contain CCA and non-tumor control groups; both the serum and tissue data of patients with CCA were included in this study, but cell line data were excluded. Secondly, in terms of sample size, each dataset should contain at least three samples. Thirdly, the raw expression data of mature or precursor miRNAs should be available and reprocessable. Finally, all miRNA-microarray and miRNA-sequencing data with other intervening factors were removed, such as experiments with gene knockdown and other treatments. In addition, The Cancer Genome Atlas (TCGA) cholangiocarcinoma miRNA mature strand expression RNA-sequencing data were downloaded from UCSC Xena (xena.ucsc.edu), which included 36 CCA samples and nine normal samples. The corresponding clinicopathological parameters (sex, age, smoking status, Tumor, Node and Metastasis stage, pathological stage and grade) of CCA samples were also investigated, but the clinical parameters of some samples were not available on TCGA. 


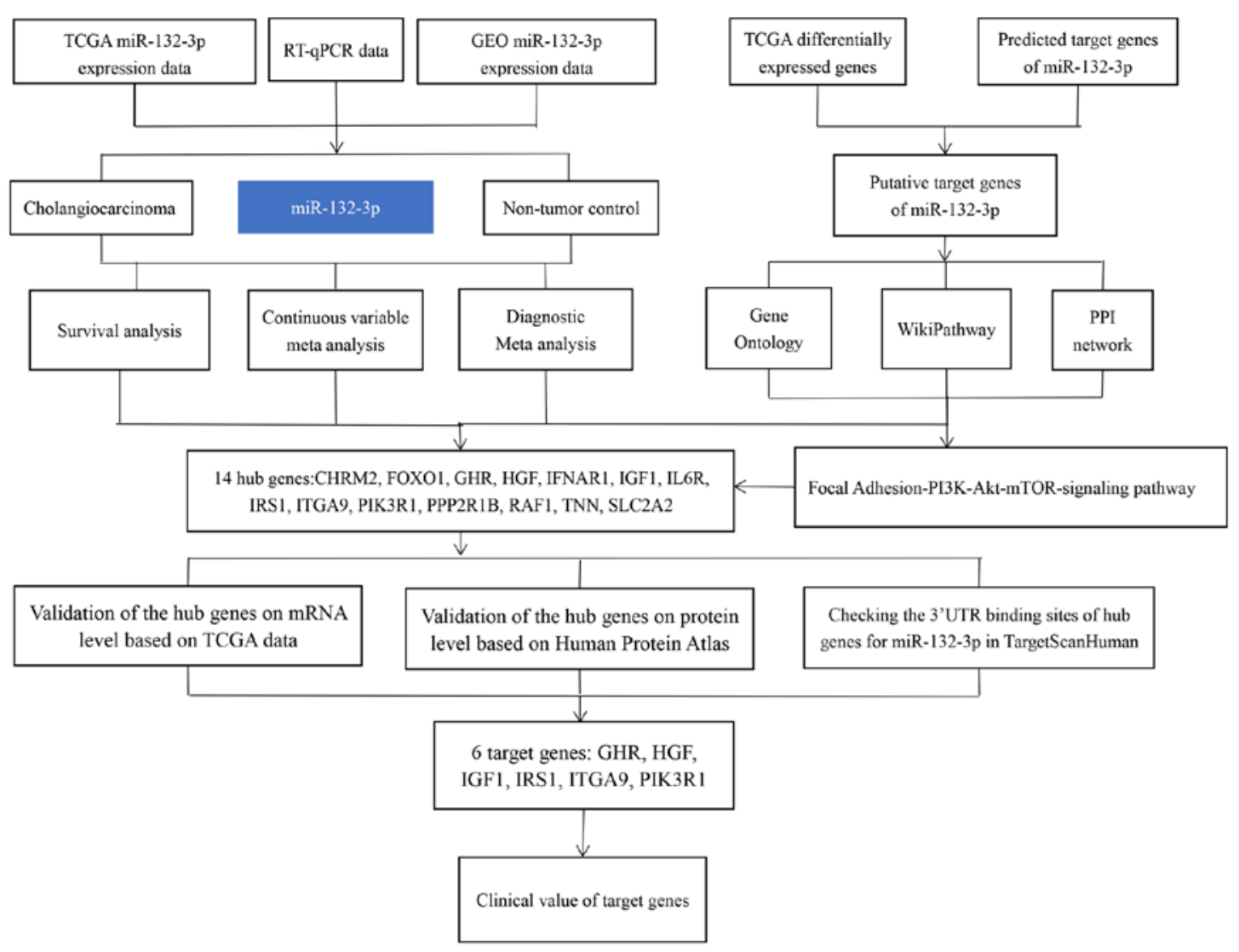

Figure 1. Flowchart representing the main design of the present study. miR-132-3p, microRNA-132-3p.

Transcripts Per Kilobase Million (TPM) was used to present RNA-sequencing results. For each sample, isoform expression for the same miRNA mature strand was combined after being $\log 2$ (total_TPM +1) transformed.

For the statistical analysis, the present study first explored the association between miR-132-3p and clinicopathological features of CCA, and the miR-132-3p expression profile data acquired from each public database and RT-qPCR data were analyzed with independent sample Student's t-test or a paired samples t-test in SPSS 22.0 (IBM Corp.). The data were presented as the means \pm standard deviation. $\mathrm{P}<0.05$ was considered to indicate a statistically significant difference. In addition, the possibility of miR-132-3p being used as a biomarker for distinguishing CCA tumor tissues from normal tissues was evaluated using a receiver operating characteristic (ROC) curve analysis. The present study also performed a continuous variable meta-analysis in Stata12.0 (StataCorp LP) to calculate the standardized mean difference (SMD). This meta-analysis initially used a fixed effect model; if heterogeneity was present, a random effect model was selected instead. The heterogeneity was evaluated with a $\chi^{2}$ test. The result would be regarded as heterogeneous if $\mathrm{P}<0.05$ or $\mathrm{I}^{2}>50 \%$. A Begg's test was performed to evaluate potential publication bias. Subsequently, a summary ROC (sROC) was conducted to combine the effect of single datasets. Diagnostic odds ratio, as well as negative and positive likelihood ratios, were also analyzed. In addition, the present study performed sROC analysis to further appraise the distinguishing ability of miR-132-3p (26-28). Thirdly, a Kaplan-Meier analysis was conducted to reveal the prognostic ability of miR-132-3p in
CCA based on TCGA data, and a log-rank test was performed to compare the survival between high and low miR-132-3p expression groups. Furthermore, to improve the accuracy of the present study, survival analysis was also conducted on the GSE53870 dataset. The GSE53870 dataset is a miRNA-related GEO dataset, which includes the survival data of 63 patients with CCA.

Analysis of differentially expressed genes based on TCGA CCA mRNA expression data. The TCGA gene expression profile data of CCA and non-tumor samples were downloaded from UCSC Xena, and differentially expressed genes [defined as those with a log (fold change) equal to 1 and $\mathrm{P}<0.05$ ] were analyzed using the edgeR package (29).

Analysis of target genes of miR-132-3p in CCA. Predicted target genes of miR-132-3p were obtained from miRWalk 2.0 (http:// zmf.umm.uni-heidelberg.de/apps/zmf/mirwalk2/), which included 12 online tools [TargetScan version 6.2; MicroT4; miRanda (release date; November 1st, 2010); miRBridge (release date; April 9th, 2010); PITA version 6.0; miRMap (release date; January 9th, 2013); miRNAMap version 2; PICTAR version 2; miRDB version 4.0; RNA22 version 2; RNAhybrid version 2.1 and miRWalk version 2.0] (30-33). Genes predicted by at least three databases were selected for subsequent analysis. To increase the reliability of the predicted targets, downregulated differentially expressed genes from RNA-sequencing data were considered as possible targets, and the present study only focused on this group of genes whose mRNA expression could be influenced by miR-132-3p. The 
A

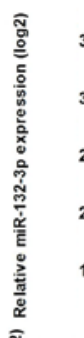

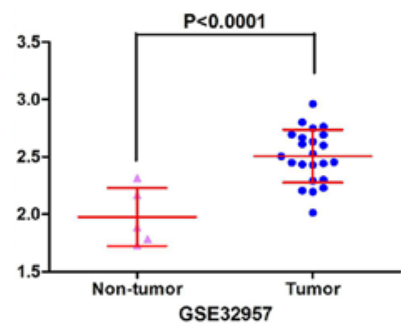

C

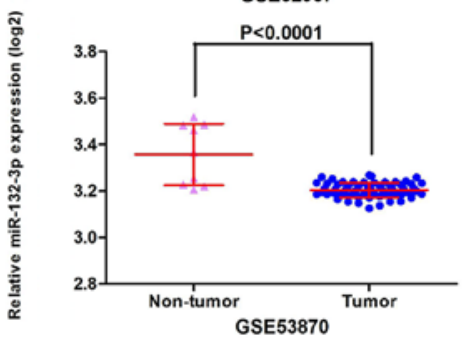

E

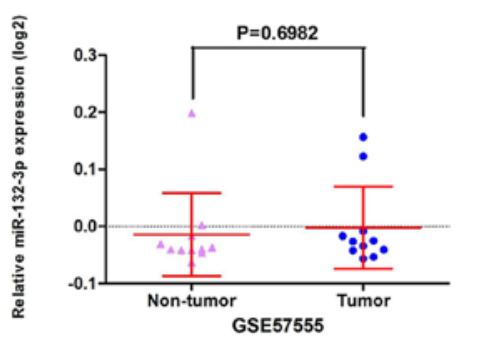

G

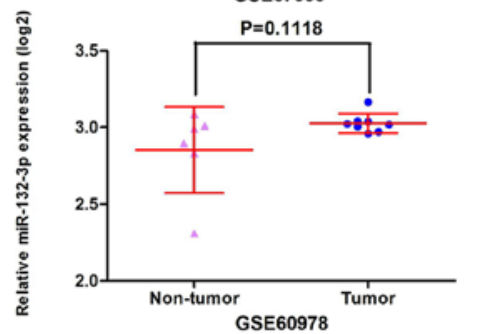

I

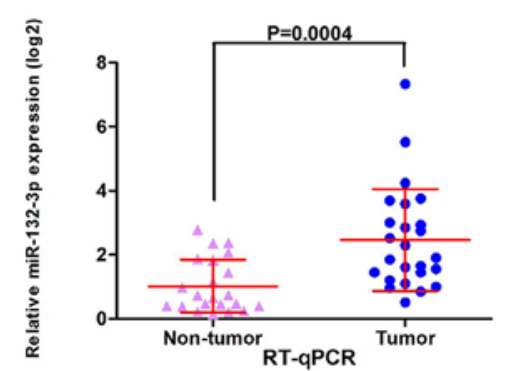

B

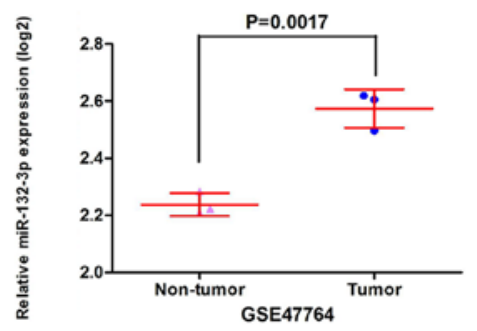

D

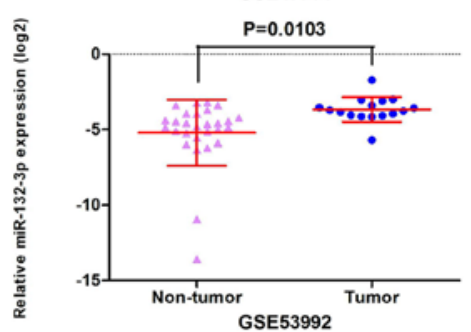

F

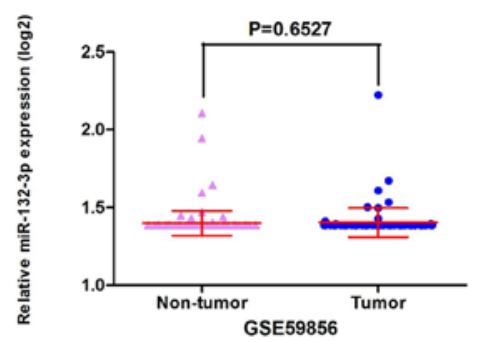

H

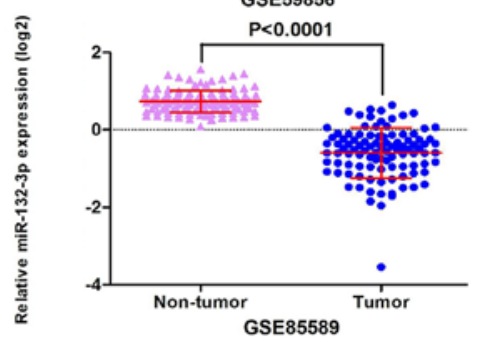

Figure 2. Scatterplots based on Gene Expression Omnibus datasets and in-house RT-qPCR analysis. (A) GSE32957, (B) GSE47764, (C) GSE53870, (D) GSE53992, (E) GSE57555, (F) GSE59856, (G) GSE60978 and (H) GSE85589 data. (I) In-house RT-qPCR analysis data. miR-132-3p, microRNA-132-3p; RT-qPCR, reverse transcription-quantitative PCR.

overlapped genes from differential expression and prediction findings were selected for further analysis.

Signaling pathway analysis of miR-132-3pin CCA.Gene Ontology (GO) annotation of the aforementioned overlapped genes was performed in WebGestalt 2017 (http://www.webgestalt.org/) (34). Pathway analysis of the aforementioned overlapped genes was also performed in WebGestalt using the WikiPathway functional database. Visualization of GO annotation was conducted using the BiNGO app of Cytoscape version 3.5.0, and protein-protein interaction (PPI) analyses were conducted in STRING (35-39).

Validation of the hub target genes at the mRNA and protein levels. TCGA mRNA expression profiles were used to validate the expression levels of hub genes at the mRNA level. The Human Protein Atlas (https://www.proteinatlas.org/) (40) was used to evaluate the protein expression levels of hub genes in CCA tissues and non-tumor intrahepatic biliary epithelium. Additionally, the binding site of miR-132-3p in hub genes was investigated with TargetScanHuman (www.targetscan.org/vert_72), and only those genes that contained 3'-untranslated region (UTR) binding sites for miR-132-3p were selected. Finally, the clinical significance of hub genes was explored by plotting ROC and survival curves.

\section{Results}

Expression of miR-132-3p in CCA. The GEO datasets (GSE32957, GSE47764 and GSE53992) indicated that 

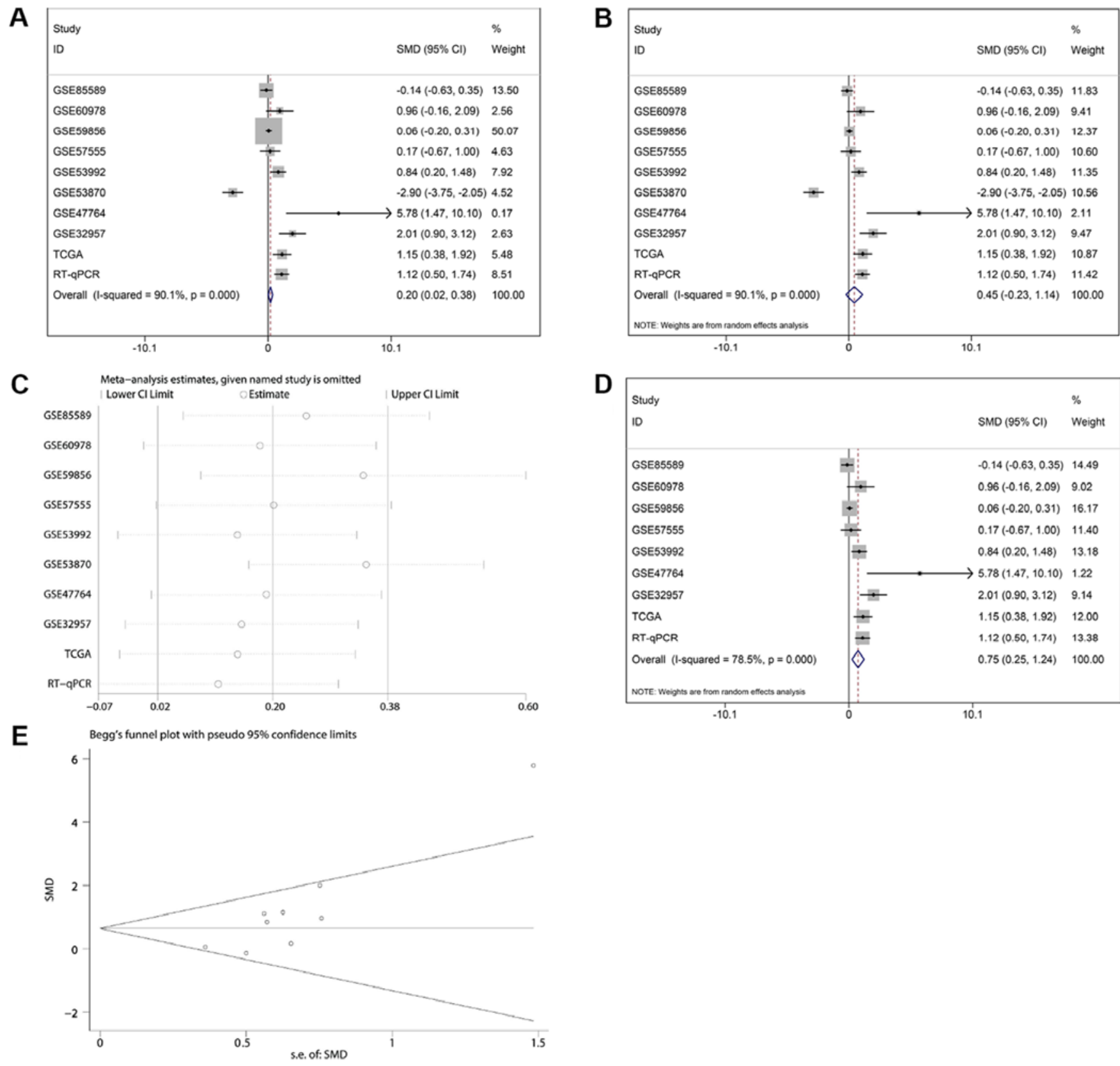

Figure 3. Continuous variable meta-analysis based on RT-qPCR, TGCA and Gene Expression Omnibus data. Forest plots based on the (A) fixed and (B) random effect models. (C) Sensitivity analysis. (D) Forest plot following exclusion of GSE53870. (E) Funnel plot. RT-qPCR, reverse transcription-quantitative PCR; TCGA, The Cancer Genome Atlas.

miR-132-3p was upregulated in CCA (Fig. 2A, B and D). Besides, according to RT-qPCR, the relative expression levels of miR-132-3p were higher in the CCA group (2.4634 \pm 1.59019$)$ compared with in non-tumor control tissues $(1.0190 \pm 0.83004$; $\mathrm{P}<0.001$; Fig. 2I). The meta-analysis contained 10 groups of data, including in-house RT-qPCR data, eight GEO miRNA-microarray datasets and one TCGA miRNA-sequencing dataset; the results revealed consistent upregulation of miR-132-3p in CCA using a random effect model (Fig. 3A and B). Regarding the SMD of miR-132-3p in CCA, heterogeneity existed with an $\mathrm{I}^{2}$ of $78.5 \%$, and GSE53870 had the greatest impact on the results of the meta-analysis based on the sensitivity analysis conducted (Fig. 3C). Therefore, a forest plot was generated following exclusion of GSE53870, which revealed an SMD of 0.75 (95\% CI: $0.25,1.24)$ (Fig. 3D and E), indicating that miR-132-3p had a general increasing trend in CCA tissues based on multiple detecting methods.

The results also indicated that miR-132-3p may have potential as a biomarker to distinguish CCA tissues from non-cancer tissues based on ROC curves of RT-qPCR, miRNA-microarray and RNA-sequencing data (Fig. 4). Moreover, the sROC revealed a consistent result with an AUC of 0.80 (95\% CI: 0.76, 0.83). The pooled sensitivity, specificity, positive likelihood ratio, negative likelihood ratio and diagnostic odds ratio of miR-132-3p in CCA tissues were 0.81 (95\% CI: 0.59, 0.93), 0.71 (95\% CI: 0.58, 0.81), 2.03 (95\% CI: 1.61, 2.54), 0.28 (95\% CI: $0.13,0.61)$ and $8.54(95 \%$ CI: 3.42, 21.30), respectively (Fig. 5B-E). 
Association between miR-132-3p and clinicopathological features of CCA. No significant association between miR-132-3p levels and survival was observed either in the miRNA-sequencing data from TCGA or GEO GSE53870 data (Fig. 6). TCGA data exhibited a similar tendency, with increased miR-132-3p expression detected in cancer tissues $(6.9586 \pm 0.52035)$ compared with in non-cancer tissues $(6.3613 \pm 0.51683 ; \mathrm{P}=0.004$; Fig. 7A and $\mathrm{B})$. Notably, the expression of miR-132-3p was significantly different in each pathological stage and grade of CCA. The expression levels of miR-132-3p in the early stage of CCA (I-II) were markedly lower than those observed in patients with advanced stage (III-IVB) $(6.8754 \pm 0.5279$ vs. $7.3034 \pm 0.3267 ; \mathrm{P}=0.003)$ (Fig. 7C and D). Consistently, miR-132-3p expression in low-grade CCA (G1-G2) was 6.7581 \pm 0.5297 , whereas in patients with high-grade CCA (G3-G4) it was 7.1191 $\pm 0.4651(\mathrm{P}=0.037$; Fig. 7E and F; Table II). We have attempted to collect more data concerning the relationships between miR-132-3p level and the progression of CCA; however, no sufficient data were available from the literature or GEO/ArrayExpress/SRA datasets.

GO and WikiPathway analysis of miR-132-3p in CCA. In total, 3,309 differentially expressed genes in CCA were identified, including 1,619 upregulated and 1,690 downregulated genes. Since miR-132-3p is highly expressed in CCA, downregulated genes are more likely to be direct targets of miR-132-3p. Due to the absence of data on the protein levels of the predicted genes, the current study focused on genes influenced by miR-132-3p at the mRNA level. In total, 555 overlapped genes were obtained, including downregulated and predicted genes (Fig. 8). The outcome of WikiPathway analysis revealed that the targets of miR-132-3p were mainly enriched in the 'Focal Adhesion-PI3 K-Akt-mTOR-signaling pathway', which is a cancer-associated pathway, and a total of 14 target genes were enriched in this pathway (Figs. 9 and 10A). In terms of GO annotation, the target genes of miR-132-3p were most significantly enriched in 'organic acid metabolic process', 'mitochondrion' and 'cofactor binding' (Figs. 10B and 11; Table III).

PPI network and validation of hub genes. The PPI network constructed in the present study is shown in Fig. 12. Genes that were enriched in the 'Focal Adhesion-PI3K-Akt-mTO R-signaling pathway' were selected as hub genes for further analysis. In total, 14 hub genes were selected, including CHRM2, FOXO1, GHR, HGF, IFNAR1, IGF1, IL6R, IRS1, ITGA9, PIK3R1, PPP2R1B, RAF1, TNN and SLC2A2. Of these, 13 genes (CHRM2, FOXO1, GHR, HGF, IGF1, IL6R, IRS1, ITGA9, PIK3R1, PPP2R1B, RAF1, TNN and SLC2A) were downregulated in CCA tissues compared with non-tumor tissues based on the TCGA and GTEx mRNA expression data (Fig. 13). These 14 hub genes were further validated at the protein expression level in The Human Protein Atlas. The results indicated that the expression levels of 10 hub genes were markedly reduced in CCA tissues compared with in normal tissues, whereas three hub genes (FOXO1, IFNAR1 and PPP2R1B) were upregulated or not differentially expressed in CCA tissues. However, the protein expression levels of IL6R in CCA tissues were not available in The Human Protein Atlas (Fig. 14). Furthermore, since the sample size was not sufficient to perform further statistical analyses, additional samples were required for verification of the present findings in future studies.

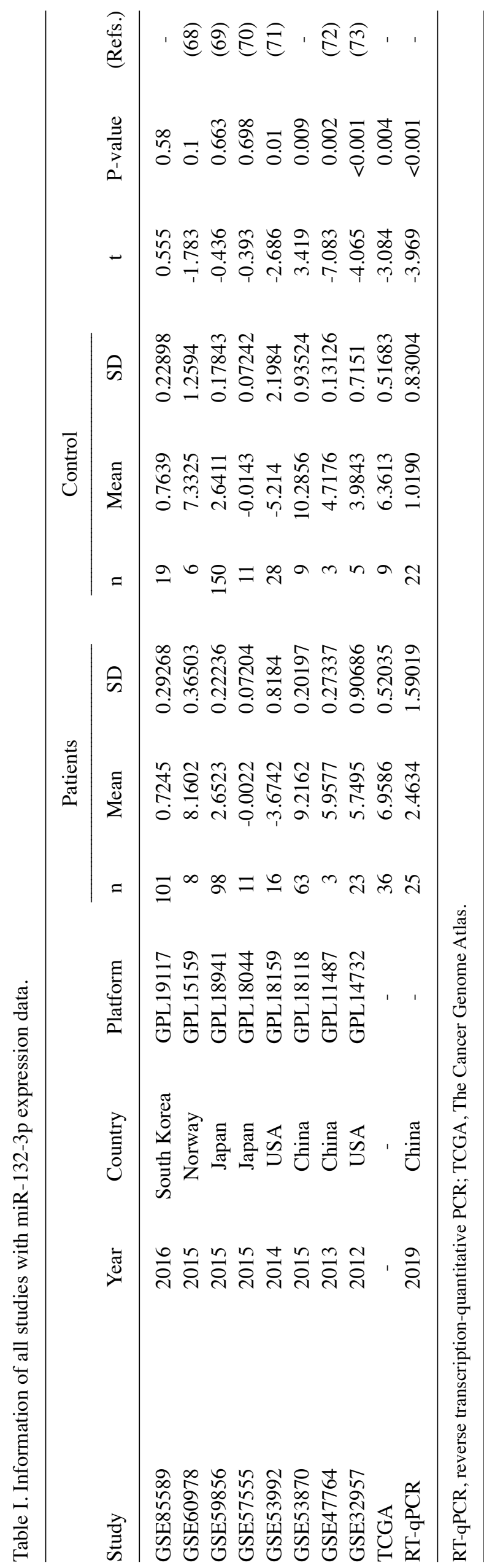


A

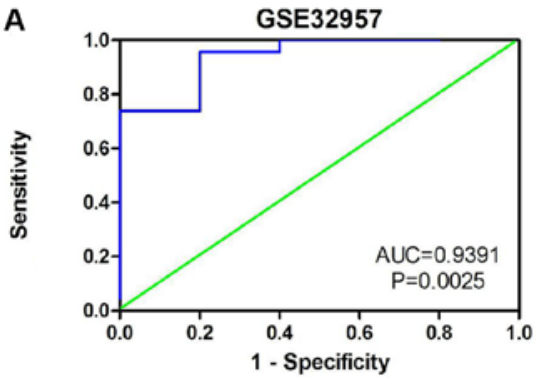

C

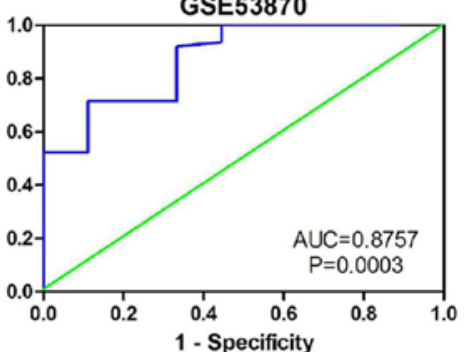

E

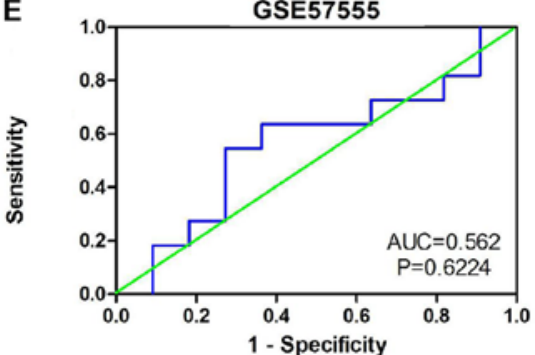

G

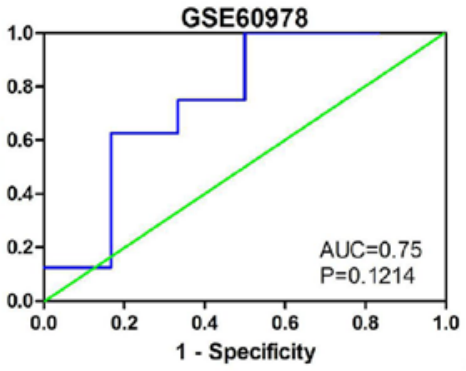

I

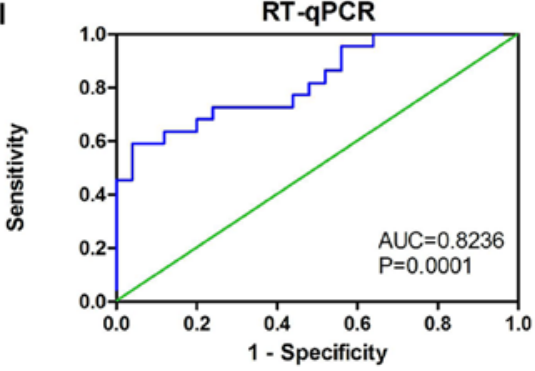

B

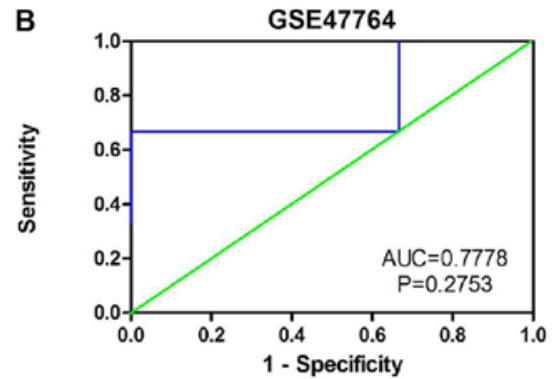

D

GSE53992

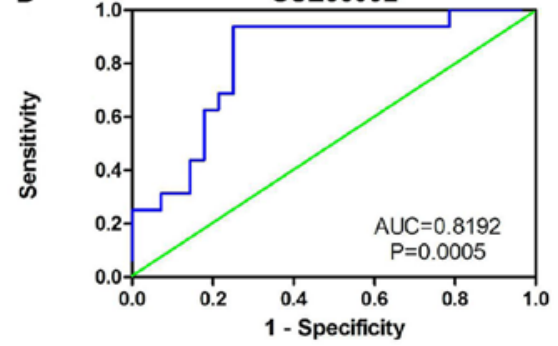

$\mathbf{F}$

GSE59856

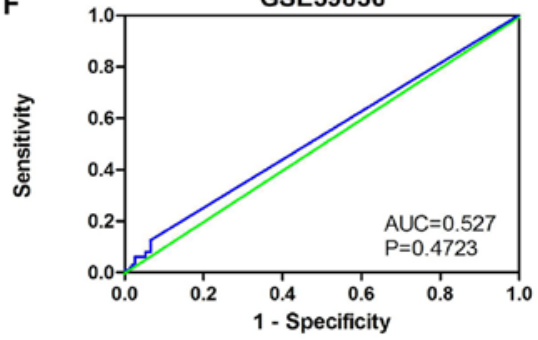

H

GSE85589

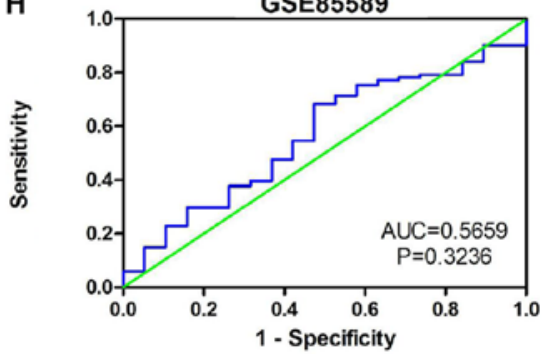

Figure 4. Receiver operating characteristic curves based on Gene Expression Omnibus datasets and in-house RT-qPCR data. (A) GSE32957, (B) GSE47764, (C) GSE53870, (D) GSE53992, (E) GSE57555, (F) GSE59856, (G) GSE60978 and (H) GSE85589 data. (I) In-house RT-qPCR analysis data. AUC, area under the curve; RT-qPCR, reverse transcription-quantitative PCR.

In the present study, only genes with low expression levels were considered potential targets of miR-132-3p in CCA. Upon evaluating the binding site of miR-132-3p in the hub genes, it was observed that miR-132-3p binds to the 3'-UTR of six hub target genes (GHR, HGF, IGF1, IRS1, ITGA9 and PIK3R1), whereas four genes (CHRM2, RAF1, TNN and SLC2A2) are not considered targets of miR-132-3p due to lacking the binding site for miR-132-3p in their 3'-UTR (Table IV). Therefore, these six hub genes with low expression levels were selected for further analysis. The ROC of these six hub genes revealed that all could serve as biomarkers to distinguish CCA tissues from normal tissues with high sensitivity and specificity (Fig. 15). However, there were no statistically significant differences in the survival analysis of these genes (Fig. 16).

\section{Discussion}

To the best of our knowledge, the expression levels and potential target genes of miR-132-3p in CCA have not been investigated to date. The present study, by combining 
A

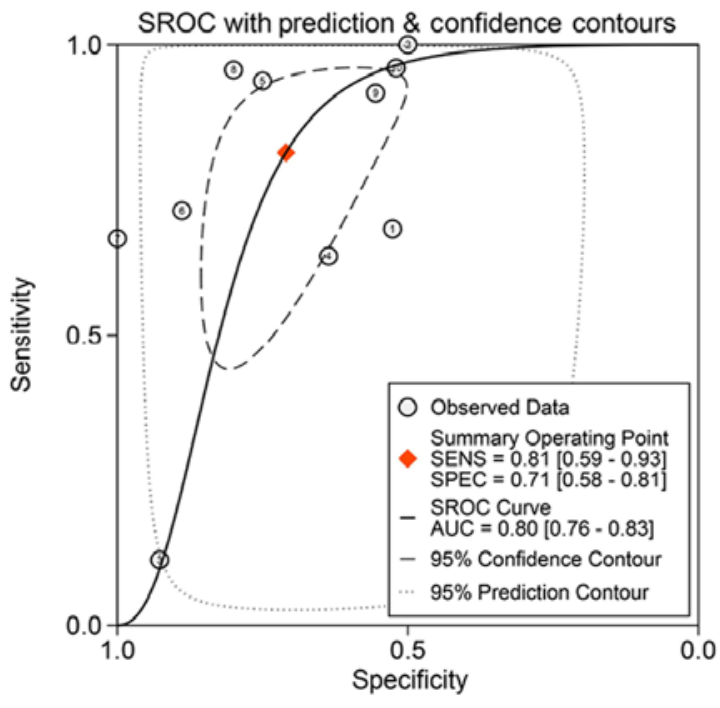

C

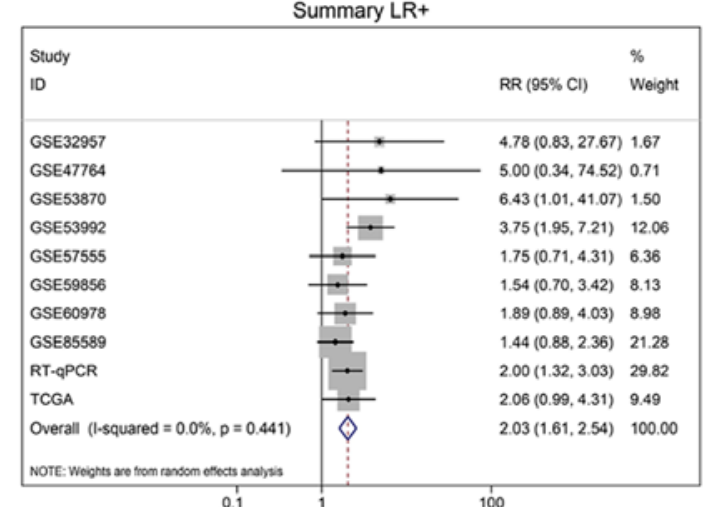

E

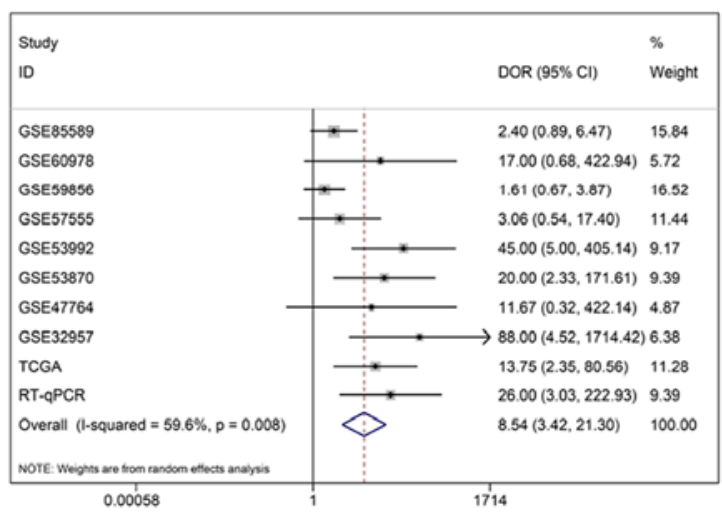

B
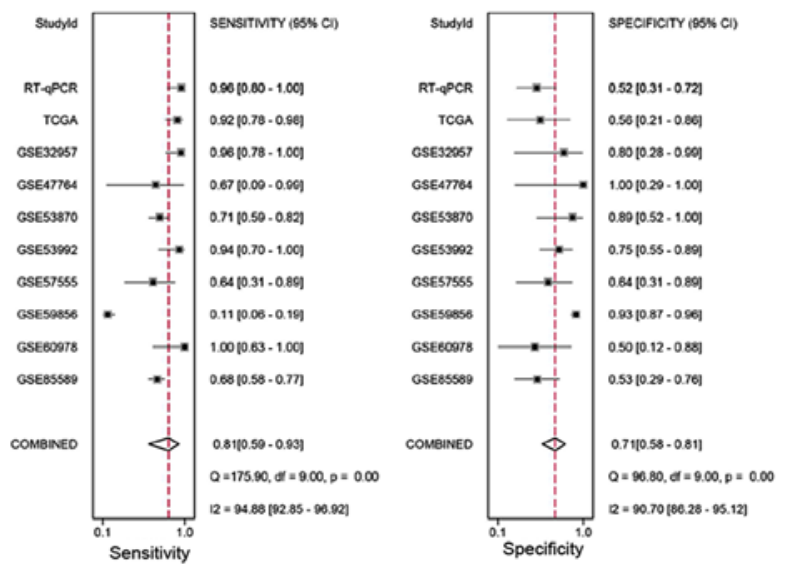

D

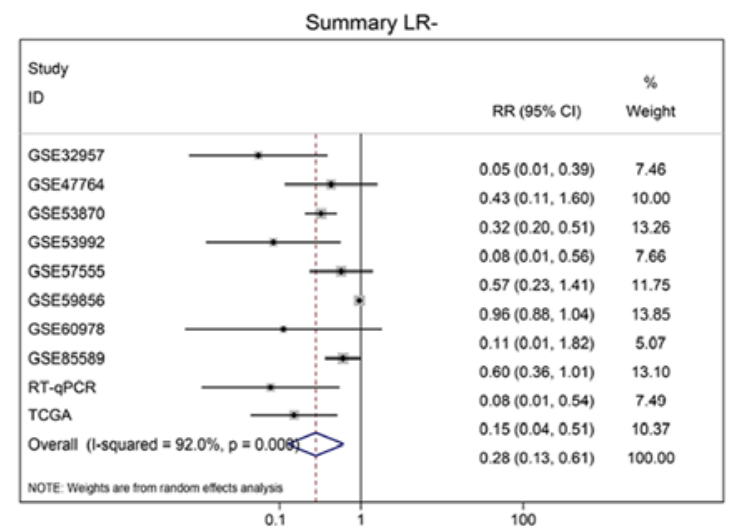

Figure 5. Validation of the ability of microRNA-132-3p to distinguish cholangiocarcinoma tissues from non-cancerous tissues. (A) sROC. (B) Pooled sensitivity and specificity. (C) Positive and negative likelihood ratios. (D) Negative likelihood ratios. (E) Forest plot of diagnostic odds ratio. sROC, summary receiver operating characteristic; RT-qPCR, reverse transcription-quantitative PCR; TCGA, The Cancer Genome Atlas.

RT-qPCR, miRNA-microarray and RNA-sequencing data, demonstrated that miR-132-3p was significantly upregulated in 321 CCA tissues compared with in 253 non-tumor controls. Furthermore, higher levels of miR-132-3p were significantly associated with CCA initiation and progression, which may be in part due to multiple target genes and signaling pathways.

miR-132-3p has been reported to be differentially expressed in numerous diseases. Notably, it is highly expressed in sural nerve biopsies from patients with neuropathy exhibiting neuropathic pain compared with those without pain (41), as well as in glioma tissues (42) and pancreatic ductal adenocarcinoma tissues (18). Conversely, miR-132-3p downregulation has been reported in the gray matter of Alzheimer's disease samples (43), mesothelioma (44) and gastric cancer tissues (19). Downregulated miR-132-3p may be a promising novel diagnostic biomarker for malignant mesothelioma with a sensitivity and specificity of 86 and $61 \%$, respectively (35). Therefore, miR-132-3p may serve distinct roles in different diseases. To the best of our knowledge, the present study is the first to detect overexpression of miR-132-3p in CCA tissues compared with in non-cancerous tissues, indicating that miR-132-3p may participate in the tumorigenesis of CCA. This phenomenon is similar to what has been documented in pancreatic ductal adenocarcinoma. Besides its function in the carcinogenesis of various types of cancer, miR-132-3p is associated with the development of tumors. A previous study reported that the single nucleotide 

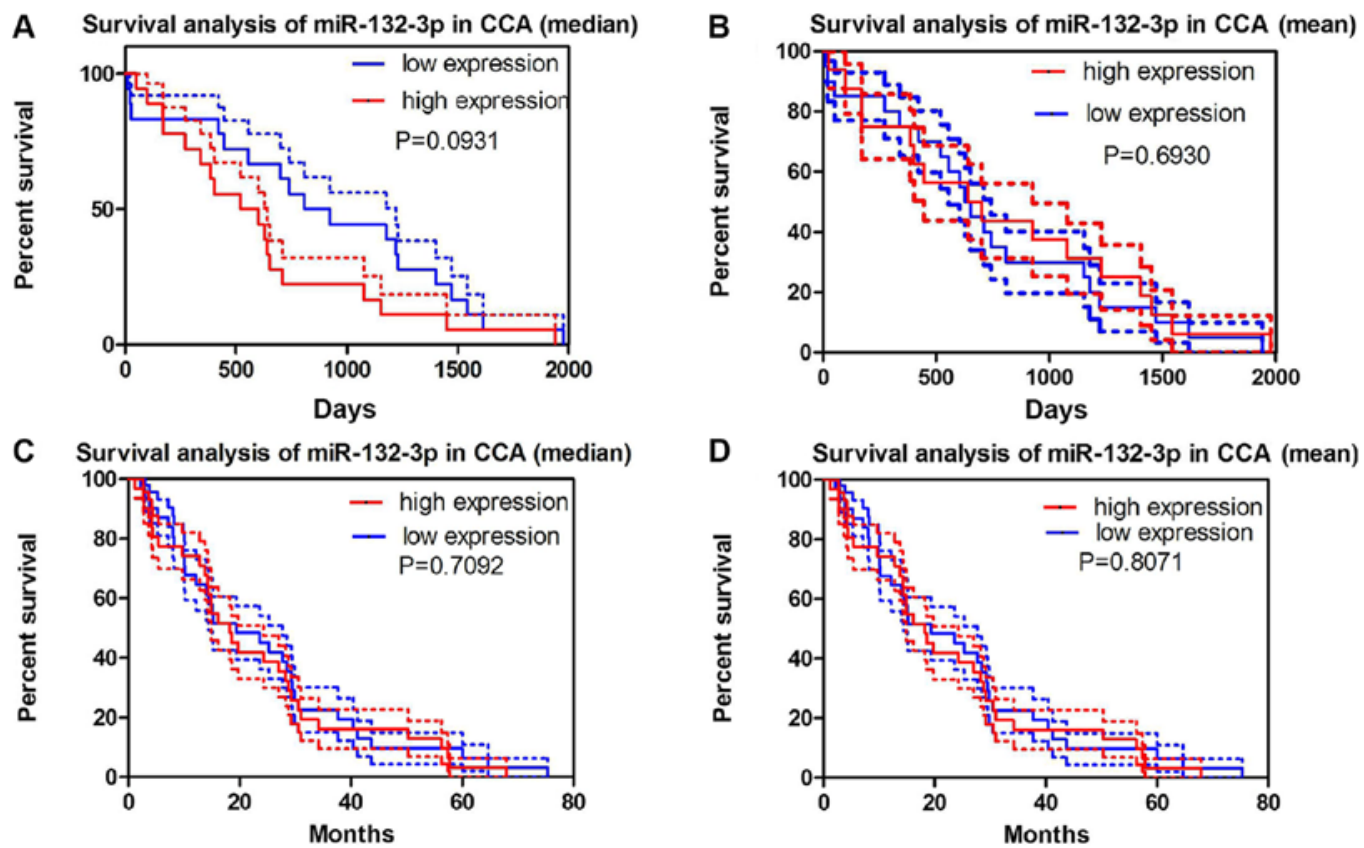

Figure 6. Survival analysis of miR-132-3p in CCA based on TCGA data and Gene Expression Omnibus GSE53870 datasets. Survival curves based on the (A) median and (B) mean expression levels of miR-132-3p in TCGA dataset. Survival curves based on the (C) median and (D) mean expression levels of miR-132-3p in the GSE53870 dataset. CCA, cholangiocarcinoma; miR-132-3p, microRNA-132-3p; TCGA, The Cancer Genome Atlas.
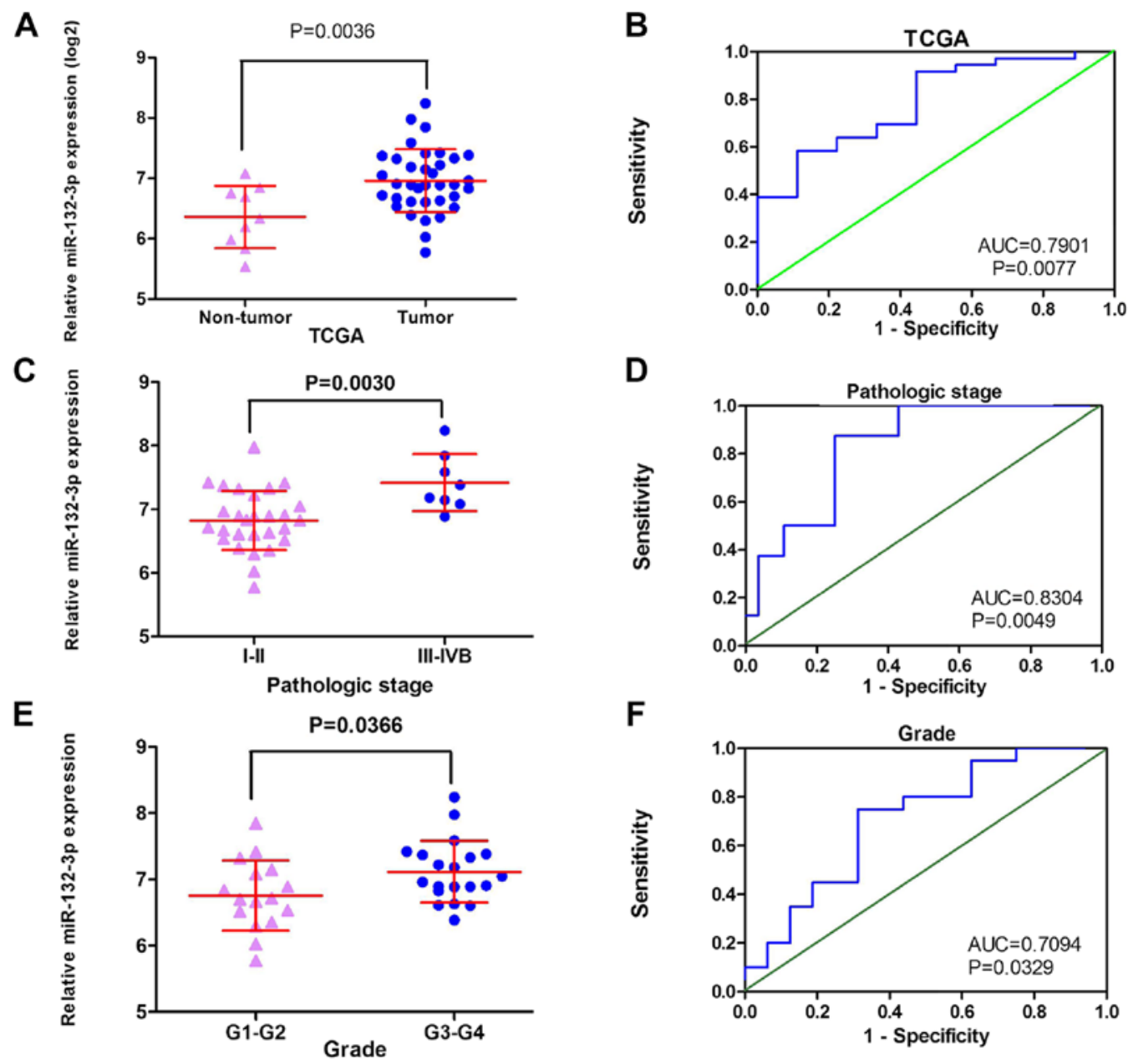

Figure 7. Association between miR-132-3p expression and pathological stage in CCA, and diagnostic value of miR-132-3p expression based on miRNA-sequencing data from TCGA. (A) Scatterplots of CCA and non-tumor tissues. (B) ROC curve analysis of CCA and non-tumor tissues. (C) Scatterplots of pathological stage. (D) ROC curve analysis of pathological stage. (E) Scatterplots of grade. (F) ROC curve analysis of grade. CCA, cholangiocarcinoma; miR-132-3p, microRNA-132-3p; ROC, receiver operating characteristic. 
Table II. Relationship between miR-132-3p expression and clinicopathological features in patients with CCA based on The Cancer Genome Atlas data.

\begin{tabular}{|c|c|c|c|c|}
\hline Clinicopathological feature & $\mathrm{n}$ & $\begin{array}{c}\text { miR-132-3p expression } \\
\log 2(\text { total_TPM }+1), \text { mean } \pm \text { standard deviation }\end{array}$ & $\mathrm{t}$ & P-value \\
\hline Tissue & & & -3.084 & 0.004 \\
\hline CCA & 36 & $6.9586 \pm 0.5204$ & & \\
\hline Non-tumor & 9 & $6.3613 \pm 0.5168$ & & \\
\hline Sex & & & -0.356 & 0.724 \\
\hline Male & 16 & $6.9236 \pm 0.5276$ & & \\
\hline Female & 20 & $6.9866 \pm 0.1177$ & & \\
\hline Age, years & & & -1.504 & 0.142 \\
\hline$<60$ & 12 & $7.1398 \pm 0.4039$ & & \\
\hline$\geq 60$ & 24 & $6.8680 \pm 0.5553$ & & \\
\hline Smoking status & & & -0.054 & 0.958 \\
\hline No & 22 & $6.9617 \pm 0.6113$ & & \\
\hline Yes & 12 & $6.9719 \pm 0.3962$ & & \\
\hline T stage & & & -1.462 & 0.153 \\
\hline $\mathrm{T} 1$ & 19 & $6.8406 \pm 0.3793$ & & \\
\hline $\mathrm{T} 2-\mathrm{T} 3$ & 17 & $7.0905 \pm 0.6289$ & & \\
\hline $\mathrm{N}$ stage & & & -1.411 & 0.167 \\
\hline No & 26 & $6.8838 \pm 0.5470$ & & \\
\hline N1-NX & 10 & $7.1532 \pm 0.4042$ & & \\
\hline M stage & & & -0.738 & 0.465 \\
\hline M0 & 28 & $6.9242 \pm 0.5332$ & & \\
\hline M1-MX & 8 & $7.0792 \pm 0.4856$ & & \\
\hline Pathological stage & & & -2.039 & 0.003 \\
\hline I-II & 29 & $6.8754 \pm 0.5279$ & & \\
\hline III-IVB & 7 & $7.3034 \pm 0.3267$ & & \\
\hline Grade & & & -2.176 & 0.037 \\
\hline G1-G2 & 16 & $6.7581 \pm 0.5297$ & & \\
\hline G3-G4 & 20 & $7.1191 \pm 0.4651$ & & \\
\hline
\end{tabular}

CCA, cholangiocarcinoma; miR-132-3p, microRNA-132-3p.

polymorphism rs1599795 in CD80 3'-UTR contributed to the occurrence of gastric cancer through disrupting the regulatory role of miR-132-3p, miR-212-3p and miR-361-5p in CD80 expression (45). Notably, in the current study, miR-132-3p upregulation was revealed to contribute to the progression of CCA, as it was closely associated with clinical stage and tumor differentiation. Therefore, miR-132-3p overexpression may lead to the occurrence of CCA and may accelerate disease progression.

The clinical role of a miRNA depends on its specific targets. Regarding the target candidates of miR-132-3p, only a few genes have been identified thus far. In breast cancer, miR-132-3p contributes to the post-transcriptional regulation of $\mathrm{BCRP} /$ ABCG2 $(8,17)$. In pancreatic ductal adenocarcinoma, overexpressed miR-132-3p may regulate executioner caspase-7 and contribute to malignant progression (18). In human osteosarcoma, miR-132-3p is regulated by the long non-coding RNA (lncRNA) taurine up-regulated 1, and may promote proliferation and suppresses apoptosis in osteosarcoma cell

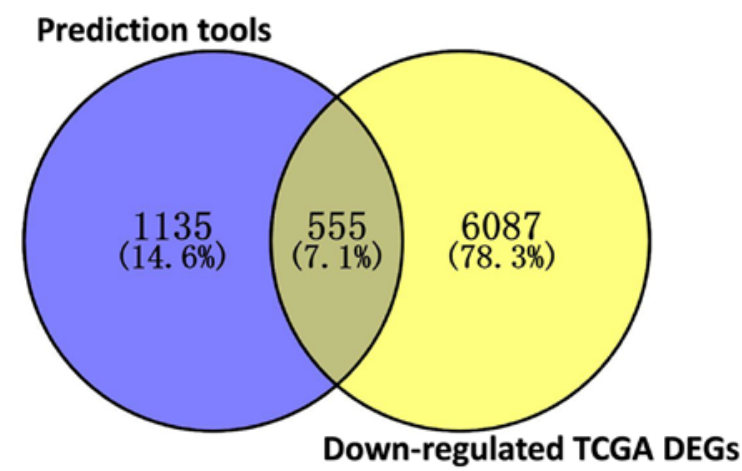

Figure 8 . Venn diagram based on the results of 12 online prediction databases and differentially expressed genes in TCGA. A total of 555 overlapped genes were subjected to further analysis. DEGS, differentially expressed genes; TCGA, The Cancer Genome Atlas.

lines (46). In colorectal cancer, miR-132-3p can be targeted by the lncRNA $X$ inactive specific transcript (47). To the best 


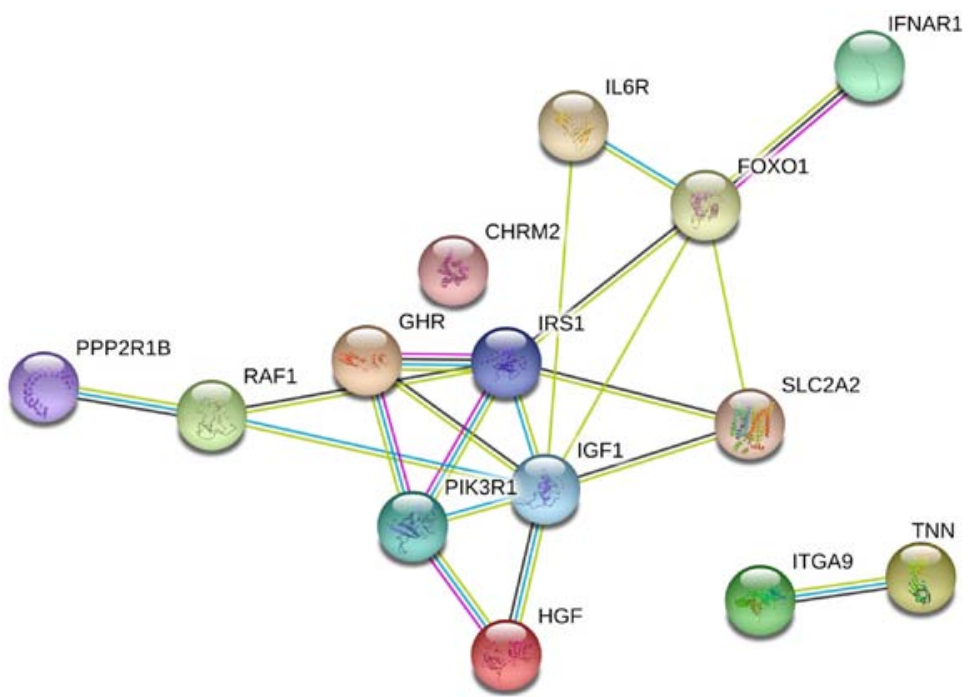

Figure 9. Protein-protein interaction network based on 14 genes involved in the 'Focal Adhesion-PI3K-Akt-mTOR-signaling pathway'.

A

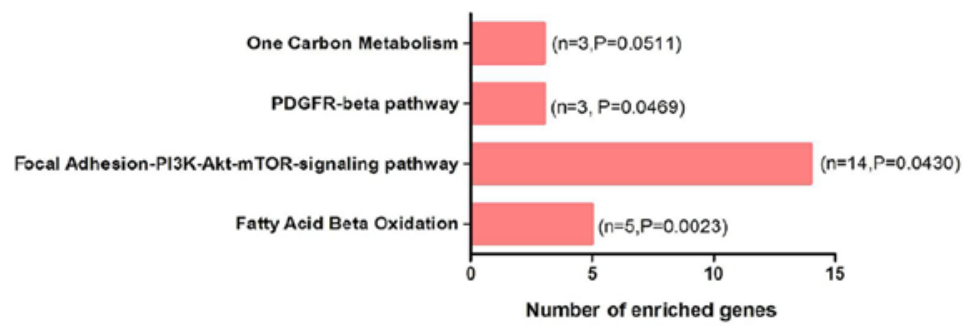

B Bar chart of Biological Process categories

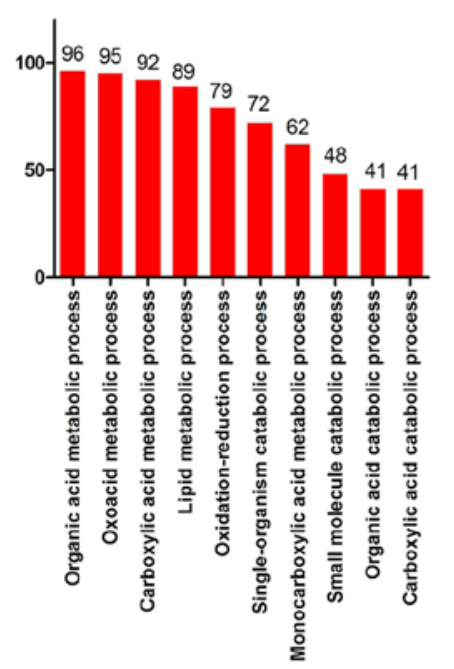

Bar chart of Cellular Component categories

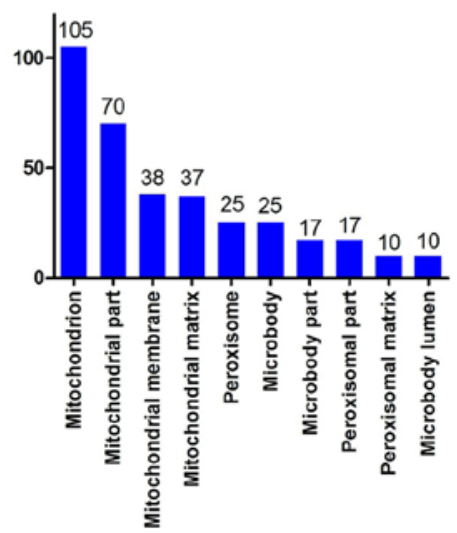

Bar chart of Molecular Function categories

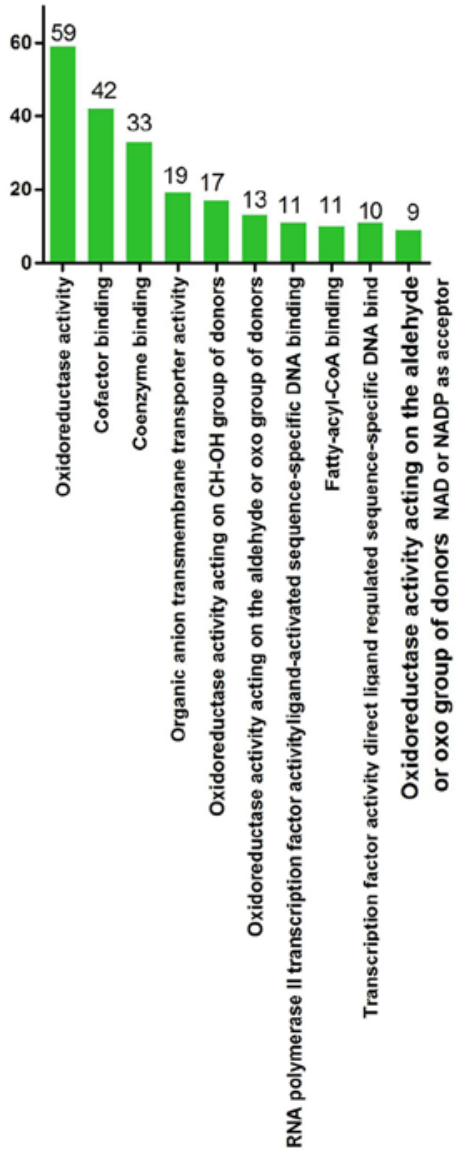

Figure 10. Bar charts of GO annotation and WikiPathway analysis of target genes of microRNA-132-3p. (A) WikiPathway analysis. (B) GO annotation analysis. GO, Gene Ontology. 

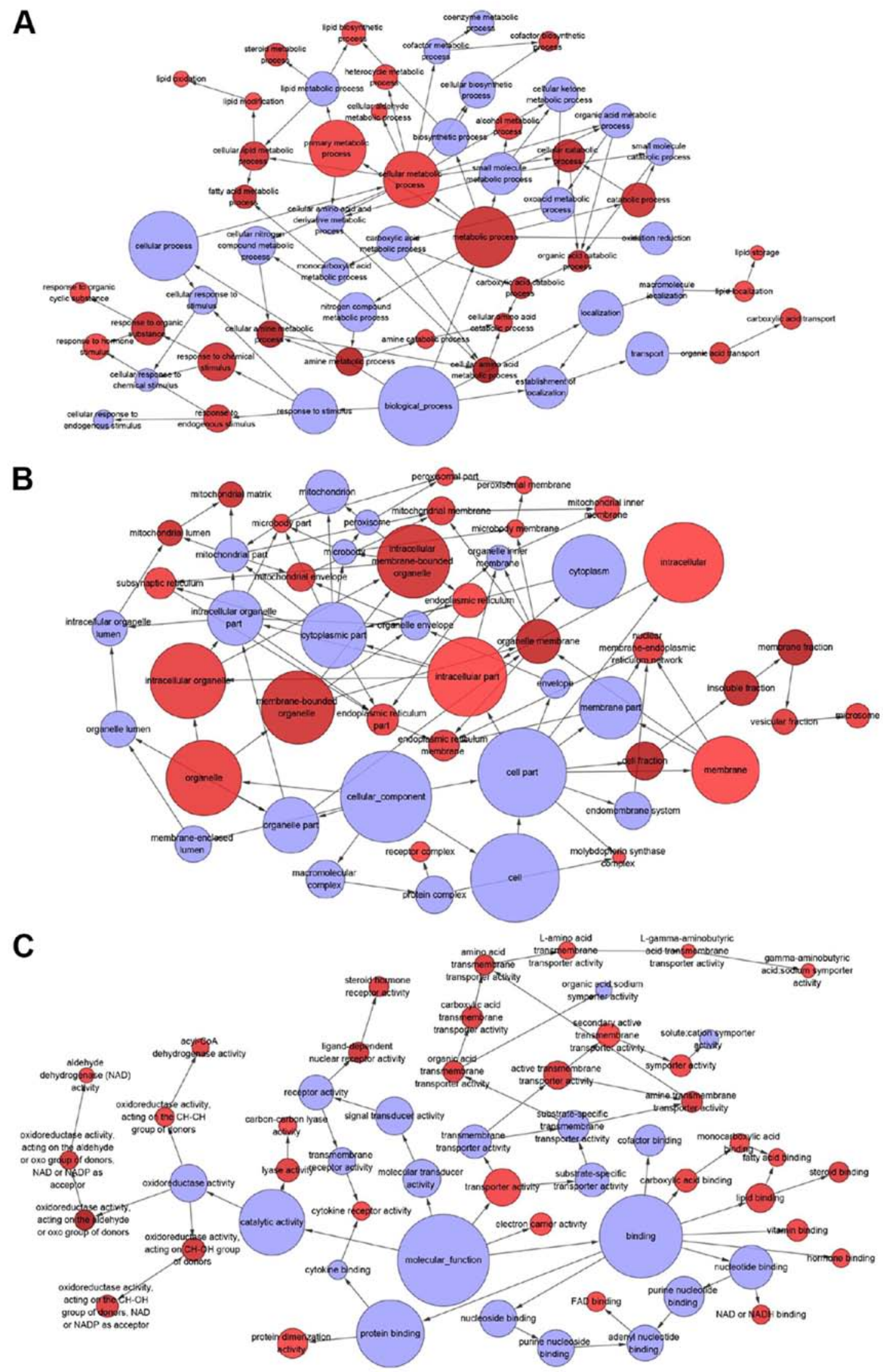

Figure 11. (A) Biological processes, (B) cellular components and (C) molecular functions were visualized using the BiNGO plugin in Cytoscape.

of our knowledge, no target gene of miR-132-3p in CCA has been studied to date. According to the outcome of bioinformatics analysis, the present study revealed that the potential target genes of miR-132-3p in CCA were principally enriched in 'Fatty Acid $\beta$ Oxidation', 'Focal Adhesion-PI3K-Akt-mT OR-signaling pathway' and 'PDGFR- $\beta$ pathway', based on 
Table III. Pathways related to microRNA-132-3p in cholangiocarcinoma.

\begin{tabular}{|c|c|c|c|}
\hline Geneset & Description & Count & P-value \\
\hline \multicolumn{4}{|l|}{ WikiPathway } \\
\hline WP143 & Fatty Acid $\beta$ Oxidation & 5 & 0.002 \\
\hline WP3932 & Focal Adhesion-PI3K-Akt-mTOR-signaling pathway & 14 & 0.043 \\
\hline WP3972 & PDGFR- $\beta$ pathway & 3 & 0.046 \\
\hline \multicolumn{4}{|c|}{ Biological process } \\
\hline GO:0006082 & Organic acid metabolic process & 96 & $<0.001$ \\
\hline GO:0016054 & Organic acid catabolic process & 41 & $<0.001$ \\
\hline GO:0019752 & Carboxylic acid metabolic process & 92 & $<0.001$ \\
\hline GO:0032787 & Monocarboxylic acid metabolic process & 62 & $<0.001$ \\
\hline GO:0043436 & Oxoacid metabolic process & 95 & $<0.001$ \\
\hline GO:0044282 & Small molecule catabolic process & 48 & $<0.001$ \\
\hline GO:0046395 & Carboxylic acid catabolic process & 41 & $<0.001$ \\
\hline GO:0055114 & Oxidation-reduction process & 79 & $<0.001$ \\
\hline GO:0044712 & Single-organism catabolic process & 72 & $<0.001$ \\
\hline GO:0006629 & Lipid metabolic process & 89 & $<0.001$ \\
\hline \multicolumn{4}{|c|}{ Cellular component } \\
\hline GO:0005739 & Mitochondrion & 105 & $<0.001$ \\
\hline GO:0044429 & Mitochondrial part & 70 & $<0.001$ \\
\hline GO:0005777 & Peroxisome & 25 & $<0.001$ \\
\hline GO:0042579 & Microbody & 25 & $<0.001$ \\
\hline GO:0005759 & Mitochondrial matrix & 37 & $<0.001$ \\
\hline GO:0044438 & Microbody part & 17 & $<0.001$ \\
\hline GO:0044439 & Peroxisomal part & 17 & $<0.001$ \\
\hline GO:0005782 & Peroxisomal matrix & 10 & $<0.001$ \\
\hline GO:0031907 & Microbody lumen & 10 & $<0.001$ \\
\hline GO:0031966 & Mitochondrial membrane & 38 & $<0.001$ \\
\hline \multicolumn{4}{|c|}{ Molecular function } \\
\hline GO:0048037 & Cofactor binding & 42 & $<0.001$ \\
\hline GO:0050662 & Coenzyme binding & 33 & $<0.001$ \\
\hline GO:0016491 & Oxidoreductase activity & 59 & $<0.001$ \\
\hline GO:0016903 & Oxidoreductase activity, acting on the aldehyde or oxo group of donors & 13 & $<0.001$ \\
\hline GO:0000062 & Fatty-acyl-CoA binding & 10 & $<0.001$ \\
\hline GO:0004879 & $\begin{array}{l}\text { RNA polymerase II transcription factor activity, ligand-activated sequence-specific } \\
\text { DNA binding }\end{array}$ & 11 & $<0.001$ \\
\hline GO:0098531 & Transcription factor activity, directligand regulated sequence-specific DNA binding & 11 & $<0.001$ \\
\hline GO:0016614 & Oxidoreductase activity, acting on $\mathrm{CH}-\mathrm{OH}$ group of donors & 17 & $<0.001$ \\
\hline GO:0016620 & $\begin{array}{l}\text { Oxidoreductase activity, acting on the aldehyde or oxo group of donors, NAD or } \\
\text { NADP as acceptor }\end{array}$ & 9 & $<0.001$ \\
\hline GO:0008514 & Organic anion transmembrane transporter activity & 19 & $<0.001$ \\
\hline
\end{tabular}

GO, Gene Ontology.

WikiPathway cancer analysis. Of these, the current study mainly focused on the 'Focal Adhesion-PI3K-Akt-mTOR-sign aling pathway', which has been reported to be associated with CCA. Focal adhesion has been reported to be involved in CCA progression and metastasis $(20,21,26,27,48-51)$, whereas the PI3K-Akt-mTOR signaling pathway serves an essential role in regulating cell survival and proliferation in unresectable and liver metastases of pancreatic cancer; notably, inhibition of the $\mathrm{PI} 3 \mathrm{~K} / \mathrm{Akt} / \mathrm{mTOR}$ signaling pathway may serve as a promising therapeutic strategy in the treatment of intrahepatic cholangiocarcinoma (52). According to previous studies, multiple molecules or drugs serve vital roles in CCA via the mTOR signaling pathway, including Fyn (53), compound C (54), sorafenib (55) and c-Myc (56). In terms of GO annotation, the 


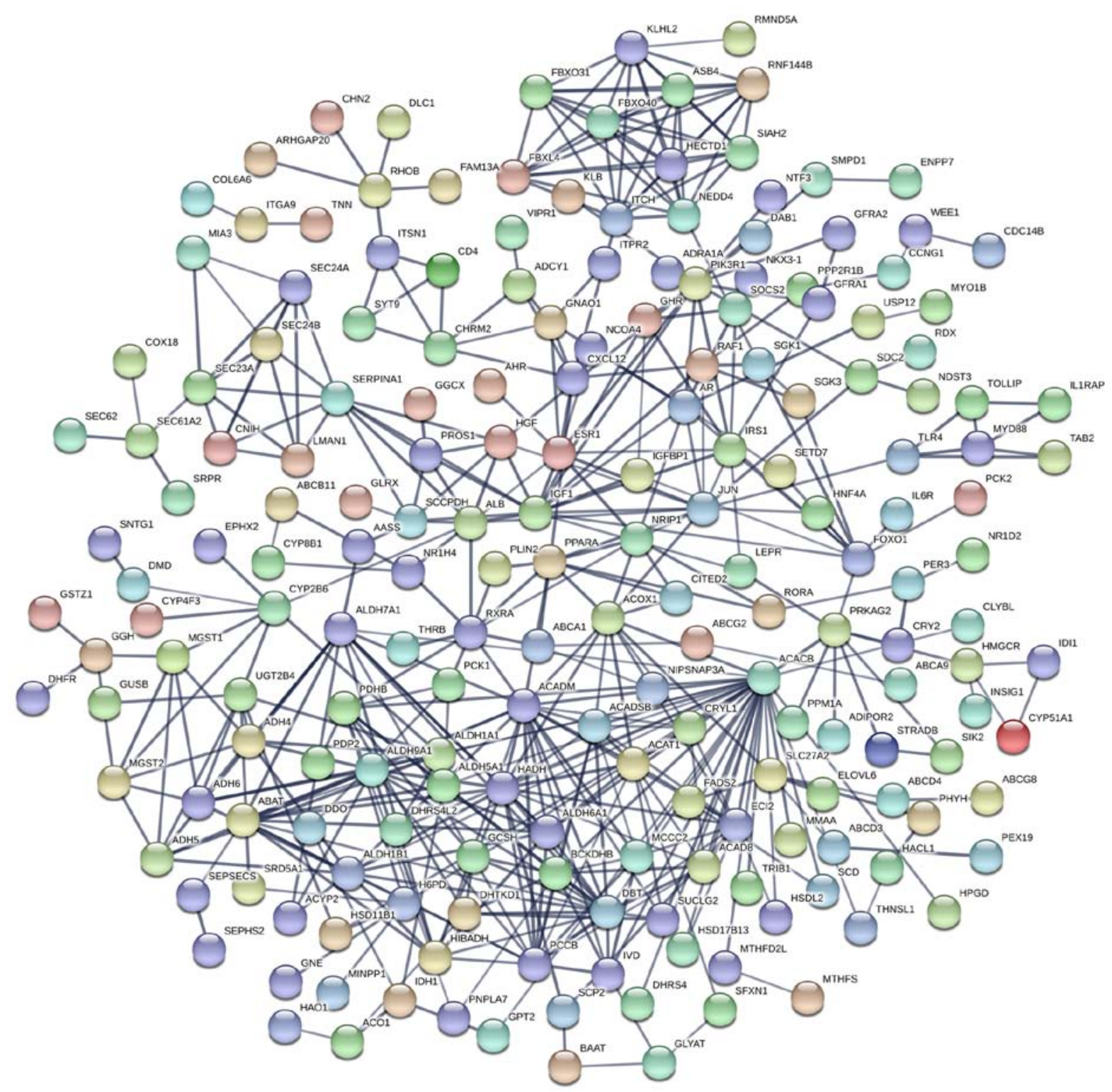

Figure 12. Protein-protein interaction of target genes. The network was performed with a medium confidence (combined score $>0.4$ ) and disconnected nodes were deleted.
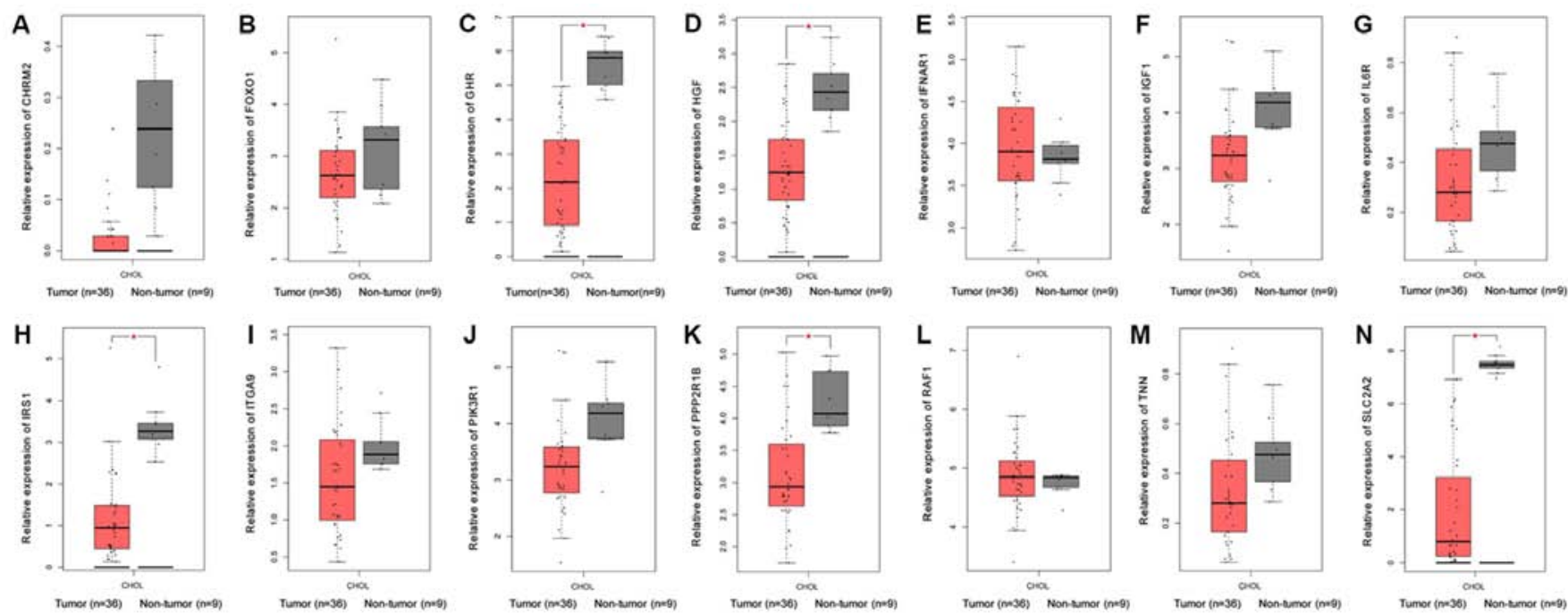

Figure 13. Validation of 14 hub genes at the mRNA level based on The Cancer Genome Atlas data. (A) CHRM2, (B) FOXO1, (C) GHR, (D) HGF, (E) IFNAR1, (F) IGF1, (G) IL6R, (H) IRS1, (I) ITGA9, (J) PIK3R1, (K) PPP2R1B, (L) RAF1, (M) TNN and (N) SLC2A2. 
A

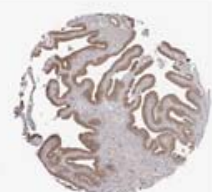

C Normal tissue

E

G

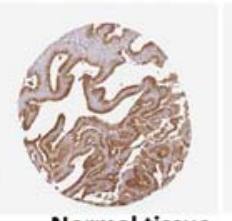

Normal tissue

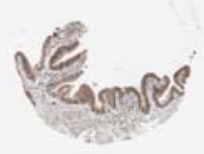

Normal tissue

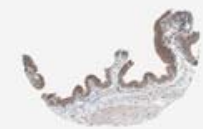

Normal tissue

I

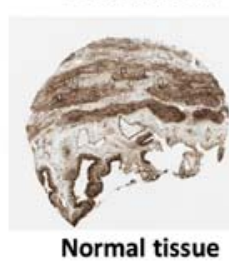

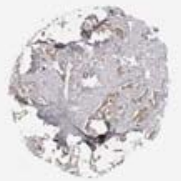

CCA tissue

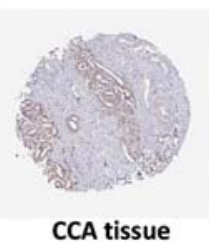

CCA tissue

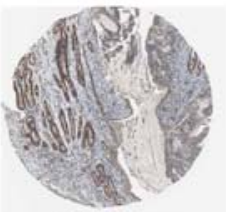

CCA tissue

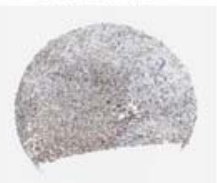

CCA tissue

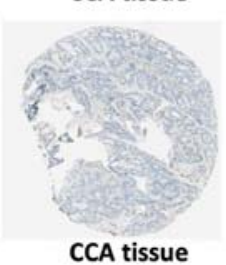

B

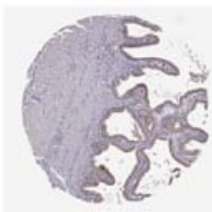

Normal tissue

D

F

$\mathrm{H}$

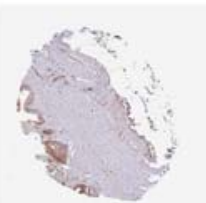

Normal tissue

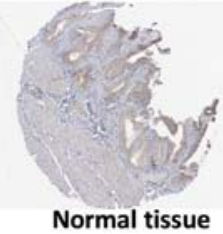

$\mathbf{J}$

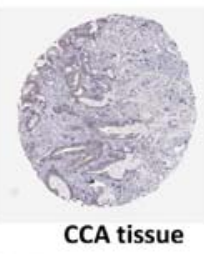

CCA tissue
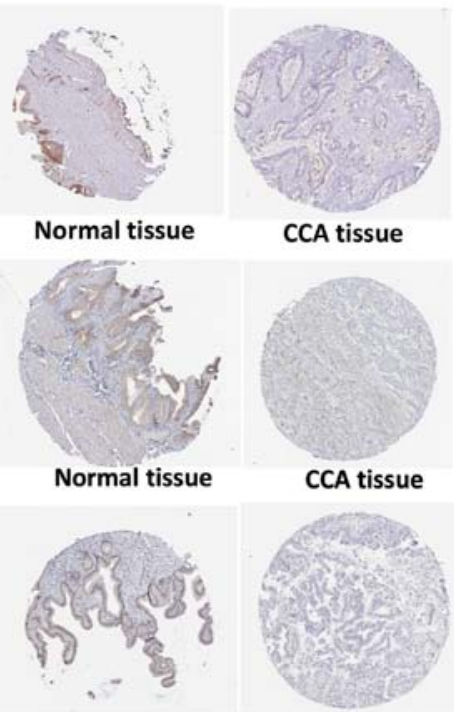

CCA tissue

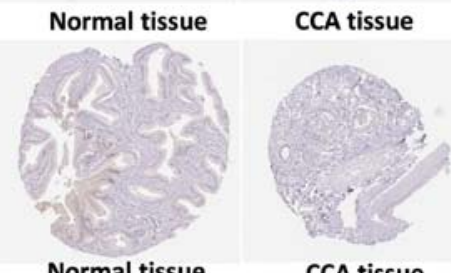

Figure 14. Validation of 10 hub genes at the protein level based on The Human Protein Atlas data. (A) CHRM2, available from: https://www.proteinatlas. org/ENSG00000181072-CHRM2/pathology/tissue/liver+cancer; https://www.proteinatlas.org/ENSG00000181072-CHRM2/tissue/gallbladder. (B) GHR, available from: https://www.proteinatlas.org/ENSG00000112964-GHR/pathology/tissue/liver+cancer; https://www.proteinatlas.org/ENSG00000112964-GHR/ tissue/gallbladder. (C) HGF, available from: https://www.proteinatlas.org/ENSG00000019991-HGF/pathology/tissue/liver+cancer; https://www.proteinatlas.org/ ENSG00000019991-HGF/tissue/gallbladder. (D) IGF1, available from: https://www.proteinatlas.org/ENSG00000017427-IGF1/pathology/tissue/liver+cancer; https://www.proteinatlas.org/ENSG00000017427-IGF1/tissue/gallbladder. (E) IRS1, available from: https://www.proteinatlas.org/ENSG00000169047-IRS1/ pathology/tissue/liver+cancer; https://www.proteinatlas.org/ENSG00000169047-IRS1/tissue/gallbladder. (F) ITGA9, available from: https://www.proteinatlas. org/ENSG00000144668-ITGA9/pathology/tissue/liver+cancer; https://www.proteinatlas.org/ENSG00000144668-ITGA9/tissue/gallbladder. (G) PIK3R1, available from: https://www.proteinatlas.org/ENSG00000145675-PIK3R1/pathology/tissue/liver+cancer; https://www.proteinatlas.org/ENSG00000145675-PIK3R1/ tissue/gallbladder. (H) RAF1, available from: https://www.proteinatlas.org/ENSG00000132155-RAF1/pathology/tissue/liver+cancer; https://www.proteinatlas.org/ENSG00000132155-RAF1/tissue/gallbladder. (I) TNN, available from: https://www.proteinatlas.org/ENSG00000120332-TNN/pathology/ tissue/liver+cancer; https://www.proteinatlas.org/ENSG00000120332-TNN/tissue/gallbladder. (J) SLC2A2, available from: https://www.proteinatlas.org/ ENSG00000163581-SLC2A2/pathology/tissue/liver+cancer; https://www.proteinatlas.org/ENSG00000163581-SLC2A2/tissue/gallbladder.

overlapped genes were principally enriched in 'organic acid metabolic process', 'mitochondrion' and 'coenzyme binding', which indicates that the targets of miR-132-3p may participate in multiple steps of the metabolism of tumor cells.

According to previously published studies, the six hub target genes identified in the current study are closely associated with numerous types of cancer. GHR has been verified to be involved in triple-negative breast cancer (57) and prostate cancer (58). HGF may participate in the regulation of neuropilin-1, and may be involved in the growth and metastasis of CCA cells (50). In addition, activation of HGF-c-MET signaling is involved in cell invasiveness and induces metastasis of biliary tract cancer (59). IGF1, which is involved in the regulation of Yes-associated protein, is associated with the progression of CCA (60). IRS1 may be a target of miR-664 and serves a role in suppressing cell proliferation and invasion in breast cancer (61). Furthermore,
IRS1 may also be targeted by miR-497, thus inhibiting the tumor growth of colorectal cancer (62). A previous study reported that upregulation of ITGA9 in response to a decrease in miR-125b levels in metastatic melanoma is responsible for melanoma tumor cell migration and invasion (63). In addition, ITGA9 is associated with other types of cancer, including nasopharyngeal carcinoma (64), breast cancer (65) and colorectal cancer (66). PIK3R1 is a target of miR-29b and miR-221, which may enhance chemosensitivity to gemcitabine in HuH28 human CCA cells (67). According to the current findings, these hub genes may be the target genes of miR-132-3p in CCA and may exert different functions; this requires further verification in future studies.

The present study has several limitations. Firstly, the sample size used for RT-qPCR testing was relatively small, which may decrease the accuracy of the conclusions. Secondly, the role of 
Table IV. Binding sites for miR-132-3p of six target genes.

\begin{tabular}{lccccc}
\hline Target & $\begin{array}{c}\text { miR-132-3p target } \\
\text { region (3'-untranslated region) }\end{array}$ & Seed match & $\begin{array}{c}\text { Context } \\
\text { score percentile }\end{array}$ & $\begin{array}{c}\text { Conserved } \\
\text { branch length }\end{array}$ & Pct \\
\hline GHR & $234-240$ & 7 mer-m8 & 95 & 5.527 & 0.54 \\
HGF & $3,387-3,393$ & 7 mer-m8 & 92 & 1.625 & $<0.1$ \\
IGF1 & $6,684-6,690$ & 7 mer-1A & 44 & 0.775 & $<0.1$ \\
IRS1 & $1,875-1,881$ & 7 mer-m8 & 65 & 2.010 & $<0.1$ \\
ITGA9 & $292-298$ & 7 mer-1A & 44 & 5.855 & 0.29 \\
PIK3R1 & $1,083-1,089$ & 7 mer-m8 & 82 & 0.154 & $<0.1$ \\
\hline
\end{tabular}

miR-132-3p, microRNA-132-2p; Pct, probability of conserved targeting.
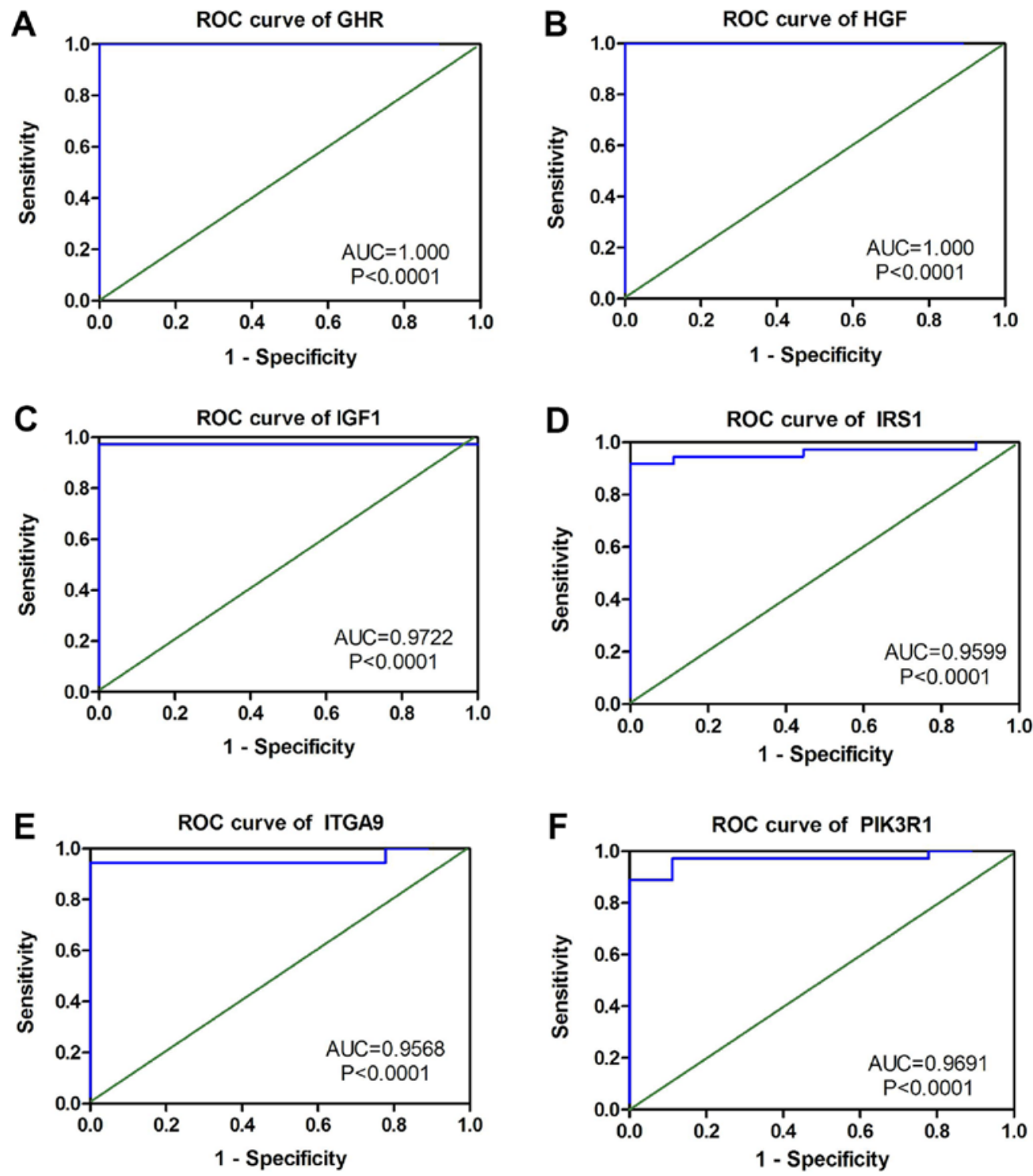

Figure 15. ROC curves of six selected genes based on The Cancer Genome Atlas. (A) GHR, (B) HGF, (C) IGF1, (D) IRS1, (E) ITGA9 and (F) PIK3R1. AUC, area under the curve; ROC, receiver operating characteristic.

miR-132-3p in progression and survival requires further study. Thirdly, the potential target genes of miRNA-132-3p were only initially verified by their expression levels; therefore, further in vitro or in vivo experiments are necessary.
In conclusion, upregulation of miR-132-3p may serve a pivotal role in the tumorigenesis and progression of CCA by targeting different pathways. Further studies are required to support the current findings. 

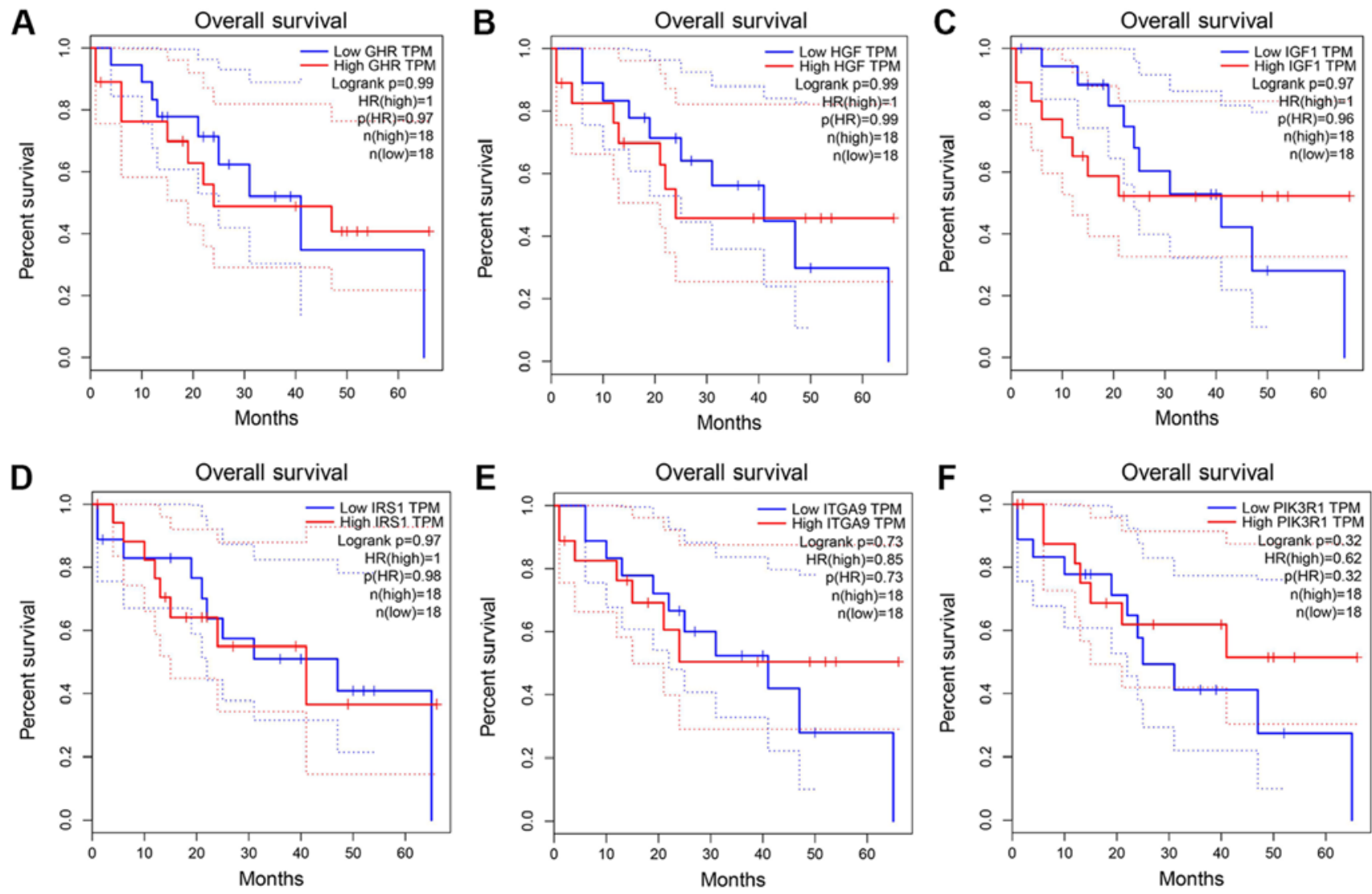

Figure 16. Survival curve analysis of six selected genes based on The Cancer Genome Atlas. (A) GHR, (B) HGF, (C) IGF1, (D) IRS1, (E) ITGA9 and (F) PIK3R1.

\section{Acknowledgements}

Not applicable.

\section{Funding}

The study was supported by the Promoting Project of Basic Capacity for Young and Middle-aged University Teachers in Guangxi (grant no. 2017KY0111), Innovation Project of Guangxi Graduate Education (grant no. YCBZ2017045) and the National Natural Science Foundation of China (grant no. 31760319).

\section{Availability of data and materials}

The datasets generated and/or analyzed during the current study are available in the TCGA (http://cancergenome.nih. gov/), the GEO (https://www.ncbi.nlm.nih.gov/geo/) and the SRA (https://www.ncbi.nlm.nih.gov/sra/) data portals. The RT-qPCR data from the present study can be acquired from the correspondence author on reasonable request.

\section{Authors' contributions}

HYW performed RT-qPCR, analyzed and interpreted data, and drafted the manuscript. SX, AGL, MDW, ZBC, YXL, YH, MJL, QPH and SLP analyzed data from microarrays and miRNA RNA-sequencing, and participated in all data processing, RT-qPCR and paper draft writing. All authors read and approved the final manuscript.

\section{Ethics approval and consent to participate}

This study was approved by the Ethics Committee of First Affiliated Hospital, Guangxi Medical University, China. Informed written consent was obtained from all patients participating in the study.

\section{Patient consent for publication}

Not applicable.

\section{Competing interests}

The authors declare that they have no competing interests.

\section{References}

1. Chng KR, Chan SH, Ng AHQ, Li C, Jusakul A, Bertrand D, Wilm A, Choo SP, Tan DMY, Lim KH, et al: Tissue microbiome profiling identifies an enrichment of specific enteric bacteria in opisthorchis viverrini associated cholangiocarcinoma. EBioMedicine 8: 195-202, 2016.

2. Verathamjamras C, Weeraphan C, Chokchaichamnankit D, Watcharatanyatip K, Subhasitanont P, Diskul-Na-Ayudthaya P, Mingkwan K, Luevisadpaibul V, Chutipongtanate S, Champattanachai $\mathrm{V}$, et al: Secretomic profiling of cells from hollow fiber bioreactor reveals PSMA3 as a potential cholangiocarcinoma biomarker. Int J Oncol 51: 269-280, 2017. 
3. Rizvi S, Khan SA, Hallemeier CL, Kelley RK and Gores GJ: Cholangiocarcinoma-evolving concepts and therapeutic strategies. Nat Rev Clin Oncol 15: 95-111, 2018.

4. Zhou G, Yang Z, Wang X, Tao R and Zhou Y: TRAIL enhances shikonin induced apoptosis through ROS/JNK signaling in cholangiocarcinoma cells. Cell Physiol Biochem 42: 1073-1086, 2017.

5. Mustafa MZ, Nguyen VH, Le Naour F, De Martin E, Beleoken E, Guettier C, Johanet C, Samuel D, Duclos-Vallee JC and Ballot E: Autoantibody signatures defined by serological proteome analysis in sera from patients with cholangiocarcinoma. J Transl Med 14: 17, 2016.

6. Su Z, Liu G, Fang T, Zhang K, Yang S, Zhang H, Wang Y, Lv Z and Liu J: Expression and prognostic value of glutamate dehydrogenase in extrahepatic cholangiocarcinoma patients. Am J Transl Res 9: 2106-2118, 2017.

7. Saha SK, Zhu AX, Fuchs CS and Brooks GA: Forty-year trends in cholangiocarcinoma incidence in the U.S.: Intrahepatic disease on the rise. Oncologist 21: 594-599, 2016.

8. Ji W, Jiao J, Cheng C and Shao J: MicroRNA-21 in the pathogenesis of traumatic brain injury. Neurochem Res 43: 1863-1868, 2018

9. Jiang X, Hu S, Liu Q, Qian C, Liu Z and Luo D: Exosomal microRNA remodels the tumor microenvironment. PeerJ 5: e4196, 2017.

10. Sun X, Wang M, Liu H and Wang J: MicroRNA-423 enhances the invasiveness of hepatocellular carcinoma via regulation of BRMS1. Am J Transl Res 9: 5576-5584, 2017.

11. Wei Q, Liu H, Ai Z, Wu Y, Liu Y, Shi Z, Ren X and Guo Z: SC1 promotes MiR124-3p expression to maintain the self-renewal of mouse embryonic stem cells by inhibiting the MEK/ERK pathway. Cell Physiol Biochem 44: 2057-2072, 2017.

12. Othman $\mathrm{N}$ and Nagoor NH: miR-608 regulates apoptosis in human lung adenocarcinoma via regulation of AKT2. Int J Oncol 51: 1757-1764, 2017.

13. Sun C, Zhu J, Wu B, Chen J, Zhu Z, Cai P, Guo W, Gu Z, Wang J and Huang S: Diagnostic and prognostic value of microRNAs in cholangiocarcinoma: A systematic review and meta-analysis. Cancer Manag Res 10: 2125-2139, 2018.

14. Huang Q, Liu L, Liu CH, You H, Shao F, Xie F, Lin XS, Hu SY and Zhang $\mathrm{CH}$ : MicroRNA-21 regulates the invasion and metastasis in cholangiocarcinoma and may be a potential biomarker for cancer prognosis. Asian Pac J Cancer Prev 14: 829-834, 2013

15. Wu YF, Li ZR, Cheng ZQ, Yin XM and Wu JS: Decrease of miR-622 expression promoted the proliferation, migration and invasion of cholangiocarcinoma cells by targeting regulation of c-Myc. Biomed Pharmacother 96: 7-13, 2017.

16. Li Z, Shen J, Chan MT and Wu WK: The role of microRNAs in intrahepatic cholangiocarcinoma. J Cell Mol Med 21: 177-184 2017.

17. Reustle A, Fisel P, Renner O, Büttner F, Winter S, Rausch S, Kruck S, Nies AT, Hennenlotter J, Scharpf M, et al: Characterization of the breast cancer resistance protein (BCRP/ ABCG2) in clear cell renal cell carcinoma. Int J Cancer 143: 3181-3193, 2018

18. Park JK, Doseff AI and Schmittgen TD: MicroRNAs targeting caspase-3 and -7 in PANC-1 cells. Int J Mol Sci 19: E1206, 2018

19. Zhang T, Liu C, Huang S, Ma Y, Fang J and Chen Y: A downmodulated microRNA profiling in patients with gastric cancer. Gastroenterol Res Pract 2017: 1526981, 2017.

20. Cai Y, Wang W, Guo H, Li H, Xiao Y and Zhang Y: miR-9-5p, miR-124-3p, and miR-132-3p regulate BCL2L11 in tuberous sclerosis complex angiomyolipoma. Lab Invest 98: 856-870, 2018

21. Zhou X, Luo D, Sun H, Qi Y, Xu W, Jin X, Li C, Lin Z and Li G: MiR-132-3p regulates ADAMTS-5 expression and promotes chondrogenic differentiation of rat mesenchymal stem cells J Cell Biochem 119: 2579-2587, 2018.

22. Livak KJ and Schmittgen TD: Analysis of relative gene expression data using real-time quantitative PCR and the 2(-Delta Delta $\mathrm{C}(\mathrm{T})$ ) method. Methods 25: 402-408, 2001.

23. Barrett T, Wilhite SE, Ledoux P, Evangelista C, Kim IF, Tomashevsky M, Marshall KA, Phillippy KH, Sherman PM, Holko M, et al: NCBI GEO: Archive for functional genomics data sets-update. Nucleic Acids Res 41: D991-D995, 2013.

24. Leinonen R, Sugawara H and Shumway M; International Nucleotide Sequence Database Collaboration: The sequence read archive. Nucleic Acids Res 39: D19-D21, 2011.

25. Parkinson H, Kapushesky M, Shojatalab M, Abeygunawardena N, Coulson R, Farne A, Holloway E, Kolesnykov N, Lilja P, Lukk M, et al: ArrayExpress-a public database of microarray experiments and gene expression profiles. Nucleic Acids Res 35: D747-D750, 2007.
26. Gan BL, He RQ, Zhang Y, Wei DM, Hu XH and Chen G: Downregulation of HOXA3 in lung adenocarcinoma and its relevant molecular mechanism analysed by RT-qPCR, TCGA and in silico analysis. Int J Oncol 53: 1557-1579, 2018.

27. Deng Y, He R, Zhang R, Gan B, Zhang Y, Chen G and Hu X: The expression of HOXA13 in lung adenocarcinoma and its clinical significance: A study based on the cancer genome atlas, oncomine and reverse transcription-quantitative polymerase chain reaction. Oncol Lett 15: 8556-8572, 2018.

28. Liang YY, Huang JC, Tang RX, Chen WJ, Chen P, Cen WL, Shi K, Gao L, Gao X, Liu AG, et al: Clinical value of miR-198-5p in lung squamous cell carcinoma assessed using microarray and RT-qPCR. World J Surg Oncol 16: 22, 2018.

29. Nikolayeva $\mathrm{O}$ and Robinson MD: edgeR for differential RNA-seq and ChIP-seq analysis: An application to stem cell biology. Methods Mol Biol 1150: 45-79, 2014.

30. Gu YL, Rong XX, Wen LT, Zhu GX and Qian MQ: miR-195 inhibits the proliferation and migration of chondrocytes by targeting GIT1. Mol Med Rep 15: 194-200, 2017.

31. Zhang N, Shen Q and Zhang P: miR-497 suppresses epithelial-mesenchymal transition and metastasis in colorectal cancer cells by targeting fos-related antigen-1. Onco Targets Ther 9: 6597-6604, 2016.

32. Chi Y, Cui J, Wang Y, Du W, Chen F, Li Z, Ma F, Song B, $\mathrm{Xu} F$, Zhao Q, et al: Interferon- $\gamma$ alters the microRNA profile of umbilical cordderived mesenchymal stem cells. Mol Med Rep 14: 4187-4197, 2016.

33. Dweep H, Gretz N and Sticht C: miRWalk database for miRNA-target interactions. Methods Mol Biol 1182: 289-305, 2014.

34. Wang J, Vasaikar S, Shi Z, Greer M and Zhang B: WebGestalt 2017: A more comprehensive, powerful, flexible and interactive gene set enrichment analysis toolkit. Nucleic Acids Res 45: W130-W137, 2017.

35. Zhang Z, Zhang G, Gao Z, Li S, Li Z, Bi J, Liu X, Li Z and Kong $C$ : Comprehensive analysis of differentially expressed genes associated with PLK1 in bladder cancer. BMC Cancer 17: $861,2017$.

36. Zeng L, Yang $\mathrm{K}$ and Ge J: Uncovering the pharmacological mechanism of astragalus salvia compound on pregnancy-induced hypertension syndrome by a network pharmacology approach Sci Rep 7: 16849, 2017.

37. Zeng L, Yang K, Liu H and Zhang G: A network pharmacology approach to investigate the pharmacological effects of Guizhi Fuling Wan on uterine fibroids. Exp Ther Med 14: 4697-4710, 2017.

38. Wang A and Zhang G: Differential gene expression analysis in glioblastoma cells and normal human brain cells based on GEO database. Oncol Lett 14: 6040-6044, 2017.

39. Su G, Morris JH, Demchak B and Bader GD: Biological network exploration with Cytoscape 3. Curr Protoc Bioinformatics 47: 8.13.1-24, 2014.

40. Colwill K, Renewable Protein Binder Working Group and Gräslund S: A roadmap to generate renewable protein binders to the human proteome. Nat Methods 8: 551-558, 2011.

41. Leinders M, Üceyler N, Pritchard RA, Sommer C and Sorkin LS: Increased miR-132-3p expression is associated with chronic neuropathic pain. Exp Neurol 283: 276-286, 2016.

42. Gu Y, Cai R, Zhang C, Xue Y, Pan Y, Wang J and Zhang Z: miR-132-3p boosts caveolae-mediated transcellular transport in glioma endothelial cells by targeting PTEN/PI3K/PKB/Src/ Cav-1 signaling pathway. FASEB J 33: 441-454, 2019.

43. Pichler S, Gu W, Hartl D, Gasparoni G, Leidinger P, Keller A, Meese E, Mayhaus M, Hampel H and Riemenschneider M: The miRNome of Alzheimer's disease: Consistent downregulation of the miR-132/212 cluster. Neurobiol Aging 50: 167 el-e167 e10, 2017.

44. Weber DG, Gawrych K, Casjens S, Brik A, Lehnert M, Taeger D, Pesch B, Kollmeier J, Bauer TT, Johnen G and Brüning T: Circulating miR-132-3p as a candidate diagnostic biomarker for malignant mesothelioma. Dis Markers 2017: 9280170, 2017.

45. Wu R, Li F, Zhu J, Tang R, Qi Q, Zhou X, Li R, Wang W, Hua D and Chen W: A functional variant at miR-132-3p, miR-212-3p, and miR-361-5p binding site in CD80 gene alters susceptibility to gastric cancer in a Chinese Han population. Med Oncol 31: 60, 2014.

46. Li G, Liu K and Du X: Long non-coding RNA TUG1 promotes proliferation and inhibits apoptosis of osteosarcoma cells by sponging miR-132-3p and upregulating SOX4 expression. Yonsei Med J 59: 226-235, 2018. 
47. Song $\mathrm{H}, \mathrm{He} \mathrm{P}$, Shao T, Li Y, Li J and Zhang Y: Long non-coding RNA XIST functions as an oncogene in human colorectal cancer by targeting miR-132-3p. J BUON 22: 696-703, 2017.

48. Phanthaphol N, Techasen A, Loilome W, Thongchot S, Thanan R, Sungkhamanon S, Khuntikeo N, Yongvanit P and Namwat N: Upregulation of TCTP is associated with cholangiocarcinoma progression and metastasis. Oncol Lett 14: 5973-5979, 2017.

49. Sae-Lao T, Luplertlop N, Janvilisri T, Tohtong R, Bates DO and Wongprasert K: Sulfated galactans from the red seaweed Gracilaria fisheri exerts anti-migration effect on cholangiocarcinoma cells. Phytomedicine 36: 59-67, 2017.

50. Zhu H, Jiang X, Zhou X, Dong X, Xie K, Yang C, Jiang H, Sun X and $\mathrm{Lu} \mathrm{J}$ : Neuropilin-1 regulated by miR-320 contributes to the growth and metastasis of cholangiocarcinoma cells. Liver Int 38 : $125-135,2018$

51. Pak JH, Bashir Q, Kim IK, Hong SJ, Maeng S, Bahk YY and Kim TS: Clonorchis sinensis excretory-secretory products promote the migration and invasion of cholangiocarcinoma cells by activating the integrin $\beta 4$-FAK/Src signaling pathway. Mol Biochem Parasitol 214: 1-9, 2017.

52. Bian JL, Wang MM, Tong EJ, Sun J, Li M, Miao ZB, Li YL, $\mathrm{Zhu} \mathrm{BH}$ and $\mathrm{Xu} \mathrm{JJ}$ : Benefit of everolimus in treatment of an intrahepatic cholangiocarcinoma patient with a PIK3CA mutation. World J Gastroenterol 23: 4311-4316, 2017.

53. Lyu SC, Han DD, Li XL, Ma J, Wu Q, Dong HM, Bai C and He Q: Fyn knockdown inhibits migration and invasion in cholangiocarcinoma through the activated AMPK/mTOR signaling pathway. Oncol Lett 15: 2085-2090, 2018.

54. Zhao X, Luo G, Cheng Y, Yu W, Chen R, Xiao B, Xiang Y, Feng C, $\mathrm{Fu} \mathrm{W}$, Duan C, et al: Compound $\mathrm{C}$ induces protective autophagy in human cholangiocarcinoma cells via Akt/mTOR-independent pathway. J Cell Biochem 119: 5538-5550, 2018.

55. Yokoi K, Kobayashi A, Motoyama H, Kitazawa M, Shimizu A, Notake T, Yokoyama T, Matsumura T, Takeoka M and Miyagawa SI: Survival pathway of cholangiocarcinoma via AKT/mTOR signaling to escape RAF/MEK/ERK pathway inhibition by sorafenib. Oncol Rep 39: 843-850, 2018.

56. Luo G, Li B, Duan C, Cheng Y, Xiao B, Yao F, Wei M, Tao Q, Feng C, Xia X, et al: cMyc promotes cholangiocarcinoma cells to overcome contact inhibition via the mTOR pathway. Oncol Rep 38: 2498-2506, 2017.

57. Girgert R, Emons G and Gründker C: Inhibition of growth hormone receptor by Somavert reduces expression of GPER and prevents growth stimulation of triple-negative breast cancer by 17ß-estradiol. Oncol Lett 15: 9559-9566, 2018.

58. Recouvreux MV, Wu JB, Gao AC, Zonis S, Chesnokova V, Bhowmick N, Chung LW and Melmed S: Androgen receptor regulation of local growth hormone in prostate cancer cells. Endocrinology 158: 2255-2268, 2017

59. Heo MH, Kim HK, Lee H, Kim KM, Lee J, Park SH, Park JO, Lim HY, Kang WK, Park YS and Kim ST: The clinical impact of c-MET over-expression in advanced biliary tract cancer (BTC). J Cancer 8: 1395-1399, 2017.

60. Pei T, Li Y, Wang J, Wang H, Liang Y, Shi H, Sun B, Yin D, Sun J, Song R, et al: YAP is a critical oncogene in human cholangiocarcinoma. Oncotarget 6: 17206-17220,2015.

61. Wu L, Li Y, Li J and Ma D: MicroRNA-664 targets insulin receptor substrate 1 to suppress cell proliferation and invasion in breast cancer. Oncol Res 27: 459-467, 2019.
62. Xu Y, Chen J, Gao C, Zhu D, Xu X, Wu C and Jiang J: MicroRNA-497 inhibits tumor growth through targeting insulin receptor substrate 1 in colorectal cancer. Oncol Lett 14: 6379-6386, 2017.

63. Zhang J, Na S, Liu C, Pan S, Cai J and Qiu J: MicroRNA-125b suppresses the epithelial-mesenchymal transition and cell invasion by targeting ITGA9 in melanoma. Tumour Biol 37: 5941-5949, 2016.

64. Nawaz I, Hu LF, Du ZM, Moumad K, Ignatyev I, Pavlova TV, Kashuba V, Almgren M, Zabarovsky ER and Ernberg I: Integrin $\alpha 9$ gene promoter is hypermethylated and downregulated in nasopharyngeal carcinoma. Oncotarget 6: 31493-31507, 2015

65. Mostovich LA, Prudnikova TY, Kondratov AG, Loginova D, Vavilov PV, Rykova VI, Sidorov SV, Pavlova TV, Kashuba VI, Zabarovsky ER and Grigorieva EV: Integrin alpha9 (ITGA9) expression and epigenetic silencing in human breast tumors. Cell Adh Migr 5: 395-401, 2011.

66. Ou J, Li J, Pan F, Xie G, Zhou Q, Huang H and Liang H: Endostatin suppresses colorectal tumor-induced lymphangiogenesis by inhibiting expression of fibronectin extra domain $\mathrm{A}$ and integrin $\alpha 9$. J Cell Biochem 112: 2106-2114, 2011.

67. Okamoto K, Miyoshi K and Murawaki Y: miR-29b, miR-205 and miR-221 enhance chemosensitivity to gemcitabine in $\mathrm{HuH} 28$ human cholangiocarcinoma cells. PLoS One 8: e77623, 2013.

68. Sandhu V, Bowitz Lothe IM, Labori KJ, Lingjærde OC, Buanes T, Dalsgaard AM, Skrede ML, Hamfjord J, Haaland T, Eide TJ, et al: Molecular signatures of mRNAs and miRNAs as prognostic biomarkers in pancreatobiliary and intestinal types of periampullary adenocarcinomas. Mol Oncol 9: 758-771, 2015.

69. Kojima M, Sudo H, Kawauchi J, Takizawa S, Kondou S, Nobumasa $\mathrm{H}$ and Ochiai A: MicroRNA markers for the diagnosis of pancreatic and biliary-tract cancers. PLoS One 10: e0118220, 2015.

70. Murakami Y, Kubo S, Tamori A, Itami S, Kawamura E, Iwaisako K, Ikeda K, Kawada N, Ochiya T and Taguchi YH: Comprehensive analysis of transcriptome and metabolome analysis in intrahepatic cholangiocarcinoma and hepatocellular carcinoma. Sci Rep 5: 16294, 2015.

71. Plieskatt JL, Rinaldi G, Feng Y, Peng J, Yonglitthipagon P, Easley S, Laha T, Pairojkul C, Bhudhisawasdi V, Sripa B, et al: Distinct miRNA signatures associate with subtypes of cholangiocarcinoma from infection with the tumourigenic liver fluke opisthorchis viverrini. J Hepatol 61: 850-858, 2014

72. Peng F, Jiang J, Yu Y, Tian R, Guo X, Li X, Shen M, Xu M, Zhu F, Shi C, et al: Direct targeting of SUZ12/ROCK2 by miR-200b/c inhibits cholangiocarcinoma tumourigenesis and metastasis. Br J Cancer 109: 3092-3104, 2013.

73. Oishi N, Kumar MR, Roessler S, Ji J, Forgues M, Budhu A, Zhao X, Andersen JB, Ye QH, Jia HL, et al: Transcriptomic profiling reveals hepatic stem-like gene signatures and interplay of miR-200c and epithelial-mesenchymal transition in intrahepatic cholangiocarcinoma. Hepatology 56: 1792-1803, 2012.

This work is licensed under a Creative Commons Attribution-NonCommercial-NoDerivatives 4.0 International (CC BY-NC-ND 4.0) License. 